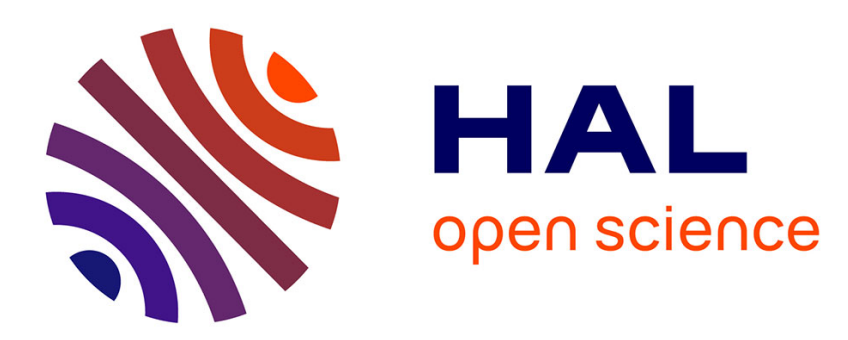

\title{
Les centons augustiniens de Florus de Lyon: minutie, érudition et vulgarisation
}

Pierre Chambert-Protat

\section{To cite this version:}

Pierre Chambert-Protat. Les centons augustiniens de Florus de Lyon: minutie, érudition et vulgarisation. Revue d'études augustiniennes et patristiques, 2014, 60 (2), pp.349-379. 10.1484/J.REA.5.107875 . halshs-01459687

\section{HAL Id: halshs-01459687 https://shs.hal.science/halshs-01459687}

Submitted on 10 Sep 2017

HAL is a multi-disciplinary open access archive for the deposit and dissemination of scientific research documents, whether they are published or not. The documents may come from teaching and research institutions in France or abroad, or from public or private research centers.
L'archive ouverte pluridisciplinaire HAL, est destinée au dépôt et à la diffusion de documents scientifiques de niveau recherche, publiés ou non, émanant des établissements d'enseignement et de recherche français ou étrangers, des laboratoires publics ou privés.

\section{(ㅇ)(1) $\$$}

Distributed under a Creative Commons Attribution - NonCommercial - NoDerivatives| 4.0 
Pierre ChAmbert-Protat, « Les centons augustiniens de Florus de Lyon : minutie, érudition et vulgarisation », dans Revue d'études augustiniennes et patristiques 60/2 (2014), p. 349-379 <halshs-01459687>

\section{Les centons augustiniens de Florus de Lyon : minutie, érudition et vulgarisation}

Outre sa grande Compilation augustinienne sur l'Apôtre en cours d'édition et désormais bien connue, Florus de Lyon ${ }^{1}$ n'a cessé de composer des opuscules à partir de sa lecture assidue et experte de l'œuvre de saint Augustin : compilations plus ou moins amples et plus ou moins élaborées, centons habilement ciselés, voire simples extraits isolés.

| Parfois très courts, de statut mal défini, généralement anonymes, vulnérables par leur nature même, les « morceaux » de ce genre restent souvent insaisissables, aussi bien pour les études des patristiciens que pour les catalogueurs de fonds manuscrits. Et si d'aventure la tradition les a quelque peu écartés du milieu intellectuel qui les a produits, il devient quasi impossible de retrouver cette origine.

\footnotetext{
${ }^{1}$ La compilation est formée de deux mille deux cent dix-huit extraits, prélevés dans toute l'œuvre de saint Augustin et agencés au fil des épîtres de saint Paul, dont ils forment un vaste commentaire ad litteram. Toutes les éditions anciennes sont insuffisantes; la première édition critique est en cours de publication. Des quatre volumes prévus, le troisième a déjà paru : Flonus LugdunENSIS, Expositio in epistolas beati Pauli ex operibus s. Augustini, Pars III : In epistolam secundam ad Corintbios. In epistolas ad Galatas, Ephesios et Philippenses, éd. P.-I. Fransen, L. De Coninck, B. Coppieters 'T Wallant, R. Demeulenaere, Turnhout, Brepols, 2011 (Corpus Christianorum Continuatio Mediaeualis, 220B.). Sur ce vaste projet, voir L. De Coninck, B. Coppieters 'T Wallant, R. DemeUlenaere, «Pour une nouvelle édition de la compilation augustinienne de Florus sur l'Apôtre », dans RBen 119 (2009), pp. 316-335. C'est cette compilation qui a donné lieu à la réinvention de Florus de Lyon dans le deuxième quart du XXe siècle, grâce aux travaux d’André WILMART ( La Collection de Bède le Vénérable sur l'Apôtre » dans RBen 38 (1926), p. 16-52 ; "Sommaire de l'Exposition de Florus sur les Épîtres ", ibid., p. 205216 ; «L'Exemplaire lyonnais de l'Exposition de Florus sur les Épîtres et ses derniers feuillets ", dans RBen 42 (1930), p. 73-76 ; « Le Mythe de Pierre de Tripoli » dans RBen 43 (1931), p. 347-352) puis de Célestin CharLIER («Les manuscrits personnels de Florus de Lyon et son activité littéraire », dans Mélanges Emmanuel Podechard. Études de sciences religieuses offertes pour son éméritat au doyen honoraire de la faculté de théologie de Lyon, Lyon, 1945, p. 71-84 ; «La Compilation augustinienne de Florus sur l'Apôtre : sources et authenticité » dans RBen 57 (1947), p. 132-186). Sur la figure de Florus de Lyon, diacre et «éminence grise » des archevêques de Lyon autour des années 825-860, on lira surtout C. ChARLIER, art. "Florus de Lyon» dans le Dictionnaire de spiritualité, t. 5, Paris, Beauchesne, 1962, col. 514-526; et désormais la monographie de K. ZECHIEL-ECKES, Florus von Lyon als Kirchenpolitiker und Publizist. Studien zur Persönlichkeit eines karolingischen "Intellektuellen 》 am Beispiel der Auseinandersetzung mit Amalarius (835-838) und des Prädestinationsstreits (851-855), Stuttgart, Jan Thorbecke, 1999 (Quellen und Forschungen zum Recht im Mittelalter, 8.).
} 
Pierre Chambert-Protat, « Les centons augustiniens de Florus de Lyon : minutie, érudition et vulgarisation », dans Revue d'études augustiniennes et patristiques 60/2 (2014), p. 349-379 <halshs-01459687>

Or, l'exceptionnelle conservation de la documentation manuscrite de Florus de Lyon permet de lui en rendre un certain nombre avec une grande certitude. En étudiant d'une part les manuscrits de travail de Florus, d'autre part son œuvre personnelle, on peut en effet étudier la façon dont Florus, le calame à la main, a élaboré son opuscule en retravaillant le texte patristique.

Deux de ces instrumenta augustiniens de Florus de Lyon ont été étudiés par Klaus ZechielEckes dans un article sur «la réception d'Augustin dans le Lyon du haut moyen âge » ${ }^{2}$. Nous avons également présenté lors d'un colloque récent, en complément d'un dossier patristique inédit, une petite pièce augustinienne liée au travail de Florus sur la prédestination ${ }^{3}$. Nous voudrions ici produire un nouveau centon augustinien et, à cette occasion, brosser le portrait du Florus de Lyon auteur de centons : sa méthode, son intention, et son rapport de théologien du haut moyen âge à l'autorité des Pères de l'Église.

\section{Le centon inédit Etenim illa cantica : un commentaire augustinien de Cant. 1, 6-7}

En exposant à l'Atelier Médio-Latin du printemps 2013 ses premiers résultats dans la quête de l'Expositio s. Ambrosii in Canticum cantoricorum ${ }^{4}$ utilisée par | Florus dans sa compilation ambrosienne sur saint Paul, Camille Gerzaguet a fait remarquer que Mannon de Saint-Oyen ${ }^{5}$ en

\footnotetext{
${ }^{2}$ K. ZECHIEL-ECKES, « Augustinus-Rezeption im frühmittelalterlichen Lyon. Ein Quellenkritischer Beitrag zu den Beati Augustini sententiae de praedestinatione et gratia Dei et de libero bominis arbitrio. Mit Edition der ungedruckten Exzerpte aus De civitate Dei (Cod. Gent 249)», dans Quellen, Kritik, Interpretation. Festgabe zum 60. Geburtstag von Hubert Mordek, Frankfurt : Peter Lang, 1999, p. 31-56. Les B. Augustini sententiae de praedestinatione et le centon Ex libro $V$ de ciuitate Dei intéressaient particulièrement Klaus Zechiel-Eckes dans le cadre de ses recherches sur l'implication de Florus dans la querelle sur la prédestination, l'un des deux pans de sa thèse d'habilitation, publiée la même année : IDEM, Florus von Lyon... (op. cit.).

${ }^{3}$ Il s'agit des Capitula XII Pelagiana, en fait un extrait de la lettre 186 d'Augustin à Paulin de Nole, que Florus a transcrit de sa main dans le manuscrit CITTÀ DEL VATICANO, B.A.V, Vat. lat. 3852, ff. 102r-v. Nous avons montré l'usage polémique qu'il a ensuite fait de cette « fiche de lecture » au moment d'asséner à Jean Scot un dossier de neuf définitions du libre-arbitre empruntées à la Tradition. «La querelle carolingienne sur la prédestination : Histoire, textes, manuscrits », colloque organisé par Pierre CHAMBERT-PROTAT, Jérémy DELMULLe, Warren PEZÉ et Jeremy C. Thompson, et tenu à Paris les 11 et 12 octobre 2013. La publication des actes est prévue dans la Collection des Études augustiniennes, série Moyen Âge et Temps Modernes.

${ }^{4}$ Camille Gerzaguet a posé les termes du problème dans un article récent : «La Collectio ambrosienne de Florus de Lyon : sources d'une compilation et enjeux d'une méthode de travail », dans MEFRM 123/2 (2011), pp. 531-543 ; en particulier pp. 534-535.

${ }^{5}$ Mannon, qui vécut et travailla à Lyon sous l'égide de Florus, fut l'un des principaux copistes de ses œuvres : ainsi, le premier exemplaire « au propre » de la monumentale compilation augustinienne sur l'apôtre de Florus fut-il
} 
Pierre Chambert-Protat, « Les centons augustiniens de Florus de Lyon : minutie, érudition et vulgarisation », dans Revue d'études augustiniennes et patristiques 60/2 (2014), p. 349-379 <halshs-01459687>

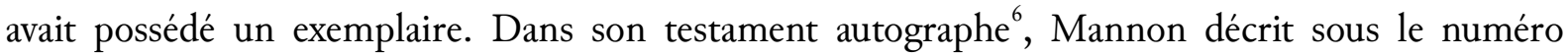
XCIII un codex aujourd'hui perdu :

XCIII. Item codex continens (1) sancti Augustini librum questionum octoginta trium. (2) Item eiusdem de decem chordis. (3) Item eiusdem sermonem de excidio urbis Romae. (4) Item eiusdem de lectione proverbiorum Salomonis : Mulierem fortem quis inveniet. (5) Item eiusdem de Eraclio presbitero successore suo. (6) Item expositio sancti Ambrosii in canticum canticorum. (7) Item quaedam sancti Augustini de eodem libro exposita. (8) Item beati Augustini questiones tres sumpte de libro questionum VIII ad Dulcitium. (9) Item orationes ex libris confessionum sancti Augustini. (10) Item capitula XII pelagiana, et quid contra eorum impietatem fides catholica teneat oppositis aliis XII capitulis expressum ex libro sancti Augustini ad Paulinum Nolanum episcopum. (11) Item ex epistola beati Leonis papae ad Turibium Asturiensem episcopum, contra priscillianitas, capitula XVI. (12) Item sententiae ex libro sancti Augustini de opere monachorum. (13) Item ex libris sancti Eucherii de variis vocabulis. (14) Item de penitentia Theodosii imperatoris. (15) Item interpretationes quorundam nominum graecorum et latinorum.

XCIII. Item, un livre contenant: (1) le livre de quatre-vingt-trois questions, de saint Augustin ; (2) du même auteur, sur les dix cordes ; (3) du même, sermon sur la chute de Rome; (4) du même, sur cette lecture tirée des Proverbes de Salomon: "Qui trouve une femme forte »; (5) du même, sur son successeur le prêtre Héraclius ; (6) Exposition sur le Cantique des cantiques, de saint Ambroise ; (7) des exposés de saint Augustin sur le même livre ; (8) trois questions extraites du livre de buit questions adressé à Dulcitius, de saint Augustin ; (9) prières tirées des Confessions, de saint Augustin ; (10) douze propositions pélagiennes et, à l'encontre de leur irréligion, la teneur de la foi catholique exprimée en douze autres contre-propositions, extraites du livre de saint Augustin adressé à Paulin de Nole ; (11) seize chapitres contre les priscillianistes, extraits de la lettre du bienheureux pape Léon à Turibius évêque d'Astorga ; (12) sentences tirées du livre sur le travail des moines, de saint Augustin ; (13) extraits des livres de saint Eucher, à propos de divers

copié intégralement de la main de Mannon dans l'actuel codex TroYEs, B.M., 96.

${ }^{6}$ Le testament a été identifié, édité et étudié par Anne-Marie TURCAN-VERKERK, « Mannon de Saint-Oyen dans l'histoire de la transmission des textes », dans RHT 29 (1999), p. 169-243. L'édition d'Anne-Marie Turcan-Verkerk est diplomatique (ibid., p. 196-203 ; l'item XCIII, p. 200-202) ; nous avons normalisé la typographie, et numéroté en chiffres arabes les textes contenus dans le codex. 
Pierre ChAmbert-Protat, « Les centons augustiniens de Florus de Lyon : minutie, érudition et vulgarisation », dans Revue d'études augustiniennes et patristiques 60/2 (2014), p. 349-379 <halshs-01459687>

mots ; (14) sur la pénitence de l'empereur Théodose ; (15) les significations de certains noms grecs et latins.

L'Expositio s. Ambrosii in Canticum cantoricorum y apparaît (notre $\mathrm{n}^{\circ} 6$ ) suivie d'un titre dont la formulation établit explicitement un rapport avec elle : quaedam sancti Augustini de eodem libro exposita. Cette remarque de Camille Gerzaguet | nous a poussé à traquer à notre tour, dans les manuscrits augustiniens de Florus de Lyon, les annotations renvoyant au Cantique des cantiques.

C'est un fait bien établi et commenté désormais que les marges des manuscrits patristiques de Florus de Lyon portent fréquemment des renvois aux épîtres pauliniennes ${ }^{7}$. Ces renvois prennent toujours la même forme : une abréviation sans équivoque du titre de l'épître, sous forme de quelques lettres semi-onciales, souvent suivies ou sommées d'une petite apostrophe très typique des annotations marginales de Florus $^{8}: \mathrm{ROM}^{\prime}, \mathrm{COR} \cdot \mathrm{I}^{\prime}, \mathrm{COR} \cdot \mathrm{II}^{\prime}, \mathrm{GAL}$ ', EPH', etc. Ce titulus minimaliste est le plus souvent surmonté d'un long tilde, et parfois précédé de «l'accolade florienne ${ }^{9}$. Ces renvois aux épîtres pauliniennes signalent des passages patristiques qui seront ensuite retranscrits en leur lieu dans les compilations patristiques sur saint Paul — dont Florus a composé au moins une quinzaine ${ }^{10}$.

\footnotetext{
${ }^{7}$ Des exemples sont analysés très précisément par C. CHARLIER, «Les manuscrits personnels... », pp. 75-78 ; IDEM, «La compilation augustinienne... », pp. 141-144; L. DE CONINCK, B. COPPIETERS 'T WALlANT, R. Demeulenaere, «Pour une nouvelle édition... », pp. 318-328; K. ZECHIEL-Eckes, « Eine neue Arbeitshandschrift des Diakons Florus von Lyon », dans RBen 119 (2009), pp. 336-370, en l'occurrence pp. 339342, 344-348 (avec quatre figures), et pp. 357-363.

${ }^{8}$ On en trouvera aisément des exemples dans les manuscrits numérisés et en ligne, tels que le manuscrit LYON, B.M., 608 (recueil augustinien : http://florus.bm-lyon.fr/visualisation.php?cote=MS0608\&folio=1) ; ou bien PARIS, B.n.F., lat. 9550 (Eucher de Lyon ; http://gallica.bnf.fr/ark:/12148/btv1b90661784/f9) ; ou encore FIRENZE, B.M.L., Plut. XIV. 21 (Ambroise de Milan; http://teca.bmlonline.it/TecaViewer/index.jsp?

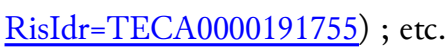

${ }^{9}$ Louis Holtz a baptisé ainsi le « signe florien par excellence : un signe fait d'un point entre deux courbes précède gloses ou références dans les marges. Ce signe est extrêmement répandu dans tous les manuscrits annotés par Florus et signale son intervention : c'est de sa part une véritable signature » (« La minuscule marginale et interlinéaire de Florus de Lyon", dans Gli autografi medievali. Problemi paleografici e filologici, Atti del convegno di studio della Fondazione Ezio Franceschini, Erice, 25 settembre - 2 ottobre 1990, Spoleto, 1994 [Quaderni di cultura mediolatina, 5], pp. 149-166, avec huit planches ; ici p. 153 ; des exemples sont reproduits dans les figures 1-23.)

${ }^{10}$ La plus grande est (1) l'Expositio in epistolas beati Pauli ex libris sancti Augustini, cf. note 1. La prétendue Collectio ex dictis XII Patrum (éditée par P.-I. Fransen, B. Coppieters 'T WALlant et R. Demeulenaere, 3 tomes, CCCM 193, 193A, 193B, Turnhout : Brepols, 2002-2007) est en réalité un groupement à peu près arbitraire de douze compilations dont chacune correspond au même projet, respectivement à partir de : (2) Cyprien de
} 
Pierre Chambert-Protat, « Les centons augustiniens de Florus de Lyon : minutie, érudition et vulgarisation », dans Revue d'études augustiniennes et patristiques 60/2 (2014), p. 349-379 <halshs-01459687>

| En réalité, ces renvois aux livres bibliques ne se limitent pas aux épîtres pauliniennes et, si

celles-ci sont largement les plus représentées, tous les livres s'y retrouvent : les Psaumes bien sûr, mais surtout les évangiles de Matthieu, puis de Jean, puis dans de moindres proportions tous les livres de l'Ancien comme du Nouveau Testament. Porter l'abréviation du livre biblique en regard d'une péricope ou d'une simple mention dans le texte patristique qu'il était en train de lire, semble relever des habitudes ordinaires de Florus de Lyon même lorsqu'il n'est pas en train de préparer une compilation.

Les renvois de Florus au Cantique des cantiques dans ses manuscrits augustiniens conservés ne sont pas légion. Trois facteurs l'expliquent : primo, Augustin lui-même ne cite pas si fréquemment le Cantique des cantiques ${ }^{11}$; secundo, nous n'avons pas conservé tous les manuscrits augustiniens de Florus, et nombre des manuscrits conservés ont été fortement endommagés dans un incendie (certainement lors du sac du quartier épiscopal de Lyon par les troupes protestantes du baron des Adrets en 1562) : il n'est pas impossible que la consomption d'amples portions des marges de gouttières en ait fait disparaitre quelques-uns ; tertio, Florus annote souvent, certes, mais pas toujours.

Les quelques renvois au Cantique que nous avons recensés ne sont pas environnés de crochets, ni de tout le travail d'emendatio et de distinctio auquel procède Florus lorsqu'il s'agit de préparer le texte pour le copiste qui devra, plus tard, mettre au propre une compilation sur l'apôtre. Rien de tel pour le Cantique chez Augustin - à une exception près.

Carthage, (3) Hilaire de Poitiers, (4) Ambroise, (5) Pacien de Barcelone, (6) Théophile d'Alexandrie, (7) Grégoire de Nazianze, (8) Ephrem le Syrien, (9) Léon le Grand, (10) Cyrille d'Alexandrie et les collections canoniques, (11) Fulgence de Ruspe, (12) Paulin de Nole et (13) Avit de Vienne. Ont été décrites mais restent à éditer deux compilations dont le propos est toujours le même : (14) Jérôme (P.-I. FRANSEN, «Description de la collection hiéronymienne de Florus de Lyon sur l'Apôtre ", dans RBen 94 (1984), pp. 195-228); et (15) Grégoire le Grand (IDEM, « Description de la collection grégorienne de Florus de Lyon sur l'Apôtre », dans RBen 98 (1988), pp. 278317). Le manuscrit PARIS, B.n.F., lat. 9550 (cf. plus haut note 8) prouve à l'évidence que Florus a élaboré une compilation (16) d'Eucher de Lyon, qui à ce jour n’a pas été retrouvée. A-t-elle seulement été menée à bien ? La compilation grégorienne est restée inachevée, mais notre liste ne prétend pas reconstituer une chronologie relative, très difficile à établir, de ces grands chantiers.

${ }^{11}$ Nous remercions le relecteur de nous avoir signalé la synthèse d'Anne-Marie LA BONNARDIÈRE, «Le Cantique des Cantiques dans l'œuvre de saint Augustin », dans REAug 1 (1955), pp. 225-238, ainsi que celle d'A. Genovese, S. Agostino e il Cantico dei Cantici. Tra esegesi e teologia, Roma, 2002. 
Pierre Chambert-Protat, « Les centons augustiniens de Florus de Lyon : minutie, érudition et vulgarisation ", dans Revue d'études augustiniennes et patristiques 60/2 (2014), p. 349-379 <halshs-01459687>

\section{Le centon de Florus dans son exemplaire d'Augustin}

Dans l'exemplaire de Florus du sermon 46 de pastoribus, dont nous avons perdu le début mais dont la fin couvre aujourd'hui les feuillets LYON, B.M., 788, ff. 67r-74v + LYON, B.M., 603, ff. $1 \mathrm{r}-\mathrm{v}$, on trouve ${ }^{12}$ un renvoi «CANT CNT' » inscrit par Florus dans la marge (f. $72 \mathrm{v}$, cf. notre planche), à la hauteur de la première citation du Cantique «Annuntia mihi quem dilexit anima mea »(Cant. 1, 6 dans AvG. serm. 46, §35 : CCSL 41, p. 560, 1. 887). Ce renvoi, de type florien ordinaire, est accompagné (un peu plus haut) d'une note de Florus dénonçant l'exégèse "stulta » des Donatistes, qu'Augustin commence ici à discuter ; puis (un peu plus bas) d'une série de crochets ouvrants et fermants, portés dans le texte et reportés dans la marge. Comme à son habitude, Florus a apporté quelques corrections au texte des passages entre crochets, et quelques précisions à | leur ponctuation. Il n'y a plus qu’à « découper selon les pointillés » pour découvrir, en concaténant les dix passages marqués, un centon formant exégèse du premier dialogue des époux (Cant. 1, 6-7).

Ce travail de préparation ne saurait être relié à celui de la Compilation augustinienne sur l'Apôtre : on ne l'y trouve pas; le centon ainsi constitué n'a rien à voir avec saint Paul, et le passage entier de saint Augustin ne s'y rapporte aucunement - Florus n'a d'ailleurs porté dans la marge aucun renvoi à une épître paulinienne. De plus, le travail de préparation est ici moins pointilleux que celui auquel se livre Florus pour la préparation de l'Expositio in epistolas b. Pauli : les corrections sont moins importantes et moins scrupuleuses; le travail de ponctuation est moins précis; et enfin tous les crochets ouvrants $(\ulcorner)$ et fermants (\urcorner$)$ sont simples. Dans les préparations de l'Expositio in epistolas b. Pauli, un crochet fermant assorti de deux points suspendus ( ('.) indique au copiste qu'il faut interrompre là la copie, mais que l'extrait ${ }^{13}$ n'est pas encore terminé : il faut chercher un peu plus loin un crochet ouvrant pourvu de deux points suspendus ( $\left({ }^{\circ}\right)$ pour reprendre là la copie interrompue ${ }^{14}$. Les préparations moins tâtillonnes du centon font figure de simples repères, à destination d'un copiste dont Florus estime avec

\footnotetext{
${ }^{12} \mathrm{Le}$ manuscrit est visible en très haute définition sur http://florus.bm-lyon.fr/visualisation.php? $\underline{\text { cote }=\text { MS0603\&folio }=72 \mathrm{~V} \_ \text {MS0788 }}$.

${ }^{13}$ Comme nous tâchons de suivre toujours attentivement le texte source de Florus d'une part, et d'autre part le traitement qu'il lui a infligé dans son œuvre personnelle, précisons immédiatement un point de terminologie : nous appellerons « extrait » (en latin excerptum) ce qui apparait dans le texte de Florus comme une citation ininterrompue d'un Père de l'Église ; et "passage » (en latin locus) ce qui apparaît comme un texte ininterrompu dans le texte source du Père de l'Église. Un extrait chez Florus correspond le plus souvent à un passage, mais il peut aussi être élaboré à partir de plusieurs.
} 
Pierre ChAmbert-Protat, « Les centons augustiniens de Florus de Lyon : minutie, érudition et vulgarisation », dans Revue d'études augustiniennes et patristiques 60/2 (2014), p. 349-379 <halshs-01459687>

confiance qu'il saura "improviser » la ponctuation et l'orthographe à mesure qu'il transcrira ; probablement lui-même.

Cet aspect général de la préparation, et le fait que nous n'avons pu trouver nulle part aucun autre extrait augustinien préparé par Florus et lié au Cantique des cantiques, nous incitent à penser que ce centon n'avait probablement pas pour destination une plus vaste compilation d'extraits d'Augustin sur le Cantique. Il a plus probablement été conçu pour rester isolé, tel quel, comme une «page » isolée de saint Augustin parmi toutes les «pages » des Pères que Florus ne cessait de verser dans ses propres dossiers pour les garder à portée de main ${ }^{15}$.

| Nous donnons ici en annexe une édition de ce centon ${ }^{16}$. Nous avons pris le parti de ne pas donner une édition diplomatique, et ce pour deux raisons. La première, c'est que le manuscrit est visible sur internet, et dans une admirable résolution : on pouvait donc privilégier ici un texte plus agréable à lire, et laisser à qui sera intéressé par les plus fines subtilités le soin de se reporter aux feuillets eux-mêmes. La seconde raison, c'est que probablement Florus aura un peu précisé la ponctuation et l'orthographe en copiant son centon au propre: il ne paraissait donc pas autrement utile de tenir un compte scrupuleux de la ponctuation et de l'orthographe un peu lâches du manuscrit-source.

On pourrait peut-être soulever quelques objections sur la légitimité de publier au long un texte reconstitué à partir d'annotations, et sans aucun témoin manuscrit conservé. Cependant, le procédé se justifie dans le cas spécifique de Florus de Lyon, puisque sa méthode d'extraction est désormais connue à la fois avec précision et pour sa précision : chaque fois que la documentation manuscrite nous conserve les préparations d'un côté et les copies contemporaines de l'autre - et

\footnotetext{
${ }^{14} \mathrm{Cf}$. les exemples très précis dont les références sont données dans notre note 7 ; mais aussi, pour des exemples ne se rapportant pas à la Compilation augustinienne sur l'Apôtre, K. ZECHIEL-ECKES, Florus von Lyon..., p. 168-9 et 215-217 (analyse de six exemples reproduits dans le texte).

${ }^{15}$ Un magnifique exemple de ces dossiers, nous est parvenu intact : le ms CitTÀ DEL VaTiCANO, B.A.V., Vat.lat. 3852, intégralement autographe, consultable en ligne dans une numérisation en haute définition : http://digi.vatlib.it/view/bav vat lat 3852. Ce dossier a été identifié et étudié par Anne-Marie TURCAN-VERKERK, «Faut-il rendre à Tertullien l'Ex libris Tertulliani de execrandis gentium diis du manuscrit Vatican latin 3852 ? I. La composition et l'origine du Vat. lat. 3852 : un dossier constitué par Florus de Lyon », dans REAug 46 (2000), pp. 205-234.

${ }^{16}$ Il est possible que le centon, tiré de son modèle et copié au propre, ait reçu un titre ; dans ce cas, suivant les habitudes de Florus en la matière, ce titre devait mentionner la source, sous une forme telle que « ex libro b. Augustini de pastoribus», et une brève description, qui évoquerait sans doute le Cantique des cantiques (« de Cantico canticorum »).
} 
Pierre CHAMBerT-Protat, « Les centons augustiniens de Florus de Lyon : minutie, érudition et vulgarisation », dans Revue d'études augustiniennes et patristiques 60/2 (2014), p. 349-379 <halshs-01459687>

le cas n'est pas rare -, la comparaison permet de constater le haut niveau de précision et de fiabilité des copies. Il n'y a pas de raison que la mise au propre de ce centon ait fait exception. Par ailleurs, un tel travail n'est pas sans exemple, puisque c'est précisément ainsi que Klaus ZechielEckes a pu reconstituer le début de la compilation de Florus De coertione iudeorum ${ }^{17}$.

Maintenant, pourrait-il s'agir là des quaedam s. Augustini de eodem libro [scil. de cantico canticorum] exposita, notre texte numéro 7 du codex XCIII du testament de Mannon de SaintOyen ? La prudence s'impose. L'Expositio Ambrosii est un florilège, tandis que la pièce qui nous occupe est un centon. On peut penser que cela les oppose nettement ; mais on peut également penser que cette différence formelle explique la différence des deux intitulés donnés par Mannon : Expositio pour un florilège, quaedam exposita pour un centon, plus bref, placé là peutêtre comme une sorte d'annexe au premier, pour la seule raison qu'il intéresse comme lui le Cantique des cantiques. Bien sûr, il n'est pas impossible que ce titre renvoie en réalité à tout autre chose : il pourrait s'agir par exemple d'un florilège augustinien sur le Cantique qui ne serait pas l'œuvre de Florus ; ou bien d'un florilège augustinien sur le Cantique réalisé par Florus, dont le centon que nous décrivons pourrait n'être qu'un des extraits... Mais nous ne connaissons rien de tel, ni | directement ni indirectement. En tout état de cause, la pièce qui nous occupe est un centon de Florus de Lyon tiré d'un passage de saint Augustin expliquant un passage du Cantique ; et à notre connaissance, c'est le seul travail de Florus sur les exégèses augustiniennes du Cantique. À l'heure actuelle, nous ne connaissons donc rien qui ressemble mieux que ce centon-là à cette rubrique-ci.

La philologie nous ayant fourni, quoi qu'il en soit, ce nouveau centon augustinien de Florus de Lyon, nous voudrions à présent tenter de comprendre le but de son auteur. Que choisit-il de retenir et d'écarter, dans l'exégèse que fait ici Augustin de Cant. 1, 6-7 ? D'autres centons du même Florus trahissent-ils la même intention ?

\section{Méthode et intention du centon en regard de sa source}

Il suffit de lire, comme s'il s'agissait de deux textes sans rapport, le texte de saint Augustin d'abord, puis le centon de Florus: la différence saute aux yeux. Revenons à l'exégèse

\footnotetext{
${ }^{17}$ K. ZECHIEL-ECKES, «Sur la tradition manuscrite des Capitula... de coertione iudeorum : ou Florus de Lyon au travail », dans RBen 107 (1997), pp. 77-87. Nous relevons en particulier cette phrase (p. 81) : «Dans le scriptorium lyonnais on obéissait presque servilement aux directives de Florus, à tel point qu'une comparaison entre le texte du Phill. 1745 [manuscrit de préparation des capitula] et celui que conserve le ms. Troyes 1406 [copie des capitula de la main de Mannon de Saint-Oyen] ne montre pas de divergences. »
} 
Pierre Chambert-Protat, « Les centons augustiniens de Florus de Lyon : minutie, érudition et vulgarisation », dans Revue d'études augustiniennes et patristiques 60/2 (2014), p. 349-379 <halshs-01459687>

augustinienne : les sermons 46 (de pastoribus) et 47 (de ouibus) constituent ensemble un long commentaire d'Ézéchiel, chapitre 34. C’est la crise donatiste qui motive, anime, traverse de part en part ces deux sermons. Leur style spontané, très oral, tout en répétitions, en parenthèses, en idées suivies, puis laissées, puis reprises, trahit l'improvisation, la parole poursuivant vivement une réflexion «en train de se faire » : à les lire, on croit voir Augustin vivant s'animer sous nos yeux. Au fil du sermon 46, nous le voyons ainsi s'engager petit à petit dans un dialogue fictif, convoquant une assemblée imaginaire de Donatistes portant arguments et contre-arguments. C'est précisément de cette façon-là qu'Augustin, suspendant Ézéchiel, en vient à consacrer une longue parenthèse ${ }^{18}$ à ces versets du Cantique. Augustin s'adresse directement aux Donatistes :

Sed recte a uobis quaeritur uox Christi, uox pastoris, quam oues audiant et sequantur. Non inuenitis quid dicatis, uocem pastoris non babetis. Audite et sequimini. Dimittite uocem lupi, sequimini uocem pastoris. Aut date uocem pastoris. 'Damus', inquiunt. Audiamus. 'Damus et nos uocem pastoris.' Audiamus. 'In canticis', inquiunt, 'canticorum loquitur sponsa ad sponsum, ecclesia ad Christum.' Nouimus cantica canticorum, sancta cantica, amatoria cantica, sancti amoris, sanctae caritatis, sanctae dulcedinis. Plane uolo inde audire uocem pastoris, uocem dulcissimi sponsi. Ede, si quid babes. Audiamus. 'Sponsa', inquiunt, 'dicit ad sponsum...' (1. 877-887)

S'il en parle, c'est donc uniquement parce que les Donatistes en parlent, et tirent argument de ce passage biblique, grâce à une exégèse fondée sur une ponctuation absurde. Augustin se livre ici à une exégèse contradictoire de l'exégèse donatiste. Il s'agit tout d'abord d'expliquer comment le texte doit être ponctué : «uerba ipsa male distinguunt...» (1. 902), "audi euidentius hac distinctione quam dicturus sum» (1.909) ; et pourquoi il ne peut en être autrement : "Puto omnes peritos imperitosque discernere genus masculinum et femininum. 'Operta', quaero cuius | generis sit. Ab omni homine quaero : Masculini est an feminini ?» (1. 914-916). C'est ainsi, à mesure qu'Augustin agrège les groupes de mots, que le dialogue de l'épouse et de l'époux se reconstitue pièce à pièce. Alors seulement (1. 929) apparaît une lecture spirituelle du passage, à travers une prosopopée de l'épouse. Mais bientôt (1. 950) Augustin revient aux affaires locales : que dira-t-on de l'interprétation donatiste selon laquelle le « midi » du dialogue des époux serait l'Afrique, et que ne dira-t-on pas de l'Église d'Afrique ! «Hic sunt sodales mali » (1. 957-958)! Et l'Église ultramarine de se plaindre au Christ : «Abundare audio haereticos in Africa, abundare

\footnotetext{
${ }^{18}$ Avg. serm. 46, §35-38 : ed. C. LAMBOT, CCSL 41, p. 560, 1. 877-1025. Dans la suite de notre propos, les citations de ce sermon renverront systématiquement à la délinéation de cette édition.
} 
Pierre ChAmbert-Protat, « Les centons augustiniens de Florus de Lyon : minutie, érudition et vulgarisation », dans Revue d'études augustiniennes et patristiques 60/2 (2014), p. 349-379 <halshs-01459687>

audio rebaptizatores in Africa... audio duas partes esse, unam partem Donati, alteram uniuerso tuo cohaerentem » (1. 960-961, 964-966). C’est alors le Christ qu'Augustin fait répondre (1. 973) et enseigner l'Église. Bien vite cependant la question du midi ramène Augustin au problème de la ponctuation (1. 983) ; puis il revient sur la question du lieu de l'Église (1. 994), cette fois d'un point de vue spirituel, le lieu étant entendu comme l'appartenance : être hérétique, c'est être dehors. Les Donatistes imaginés par Augustin voudraient alors (1. 1005) glisser un second témoignage biblique sur l'interprétation du midi ; mais Augustin leur coupe la parole pour livrer d'abord sa propre interprétation du midi, toute spirituelle : "meridiem intellego feruorem spiritalium, flagrantem igne caritatis, splendentem lumine ueritatis » (1. 1010-1011). Il finit par insister une dernière fois sur les absurdités auxquelles conduit l'identification du midi et de l'Afrique. Le Donatiste imaginaire parvient alors (1. 1025-1027) à glisser son second témoignage (Habac. 3, 3) : Augustin en a fini avec le Cantique ${ }^{19}$. Le texte, animé par les prises de paroles des Donatistes imaginaires, ne cesse donc de ricocher d'une question d'interprétation contestée à une autre : rares et brefs sont les passages où se fait jour une interprétation spirituelle et positive du dialogue de Cant. 1, 6-7.

Le texte de Florus, quant à lui, est tout entier spirituel, et les Donatistes y sont insoupçonnables. Florus s'est avant tout employé à débarrasser le texte de toutes ses références et allusions au contexte de la polémique anti-donatiste. Il supprime la mention de Donat (entre les passages e et $\mathrm{f}$ ) et du rebaptême $(\mathrm{f} / \mathrm{g})$, les prosopopées des Donatistes (avant a ; $\mathrm{i} / \mathrm{j}$ ), les disputes sur la ponctuation $(\mathrm{a} / \mathrm{b} ; \mathrm{b} / \mathrm{c} ; \mathrm{h} / \mathrm{i})$, les interprétations donatistes du midi (d/e ; après $\mathrm{j})$. En fait, ce seul parti-pris suffit à expliquer tous les découpages, sauf un : quelques mots sont écartés entre | les passages g et h, parce qu'Augustin y convoquait deux mots d'Ézéchiel 34, parvenant ainsi à se raccrocher au contexte général du sermon de pastoribus. Écarter cette allusion, c'est donc encore détacher l'exégèse de Cant. 1, 6-7 de tout ce qui l'a amenée.

\footnotetext{
${ }^{19}$ Les marginalia floriens du manuscrit de préparation nous montrent d'ailleurs un Florus qui, autour de sa préparation du centon et outre diverses notes de lecture, suit avec attention les différentes étapes du débat. En face de «Ede mihi unam Scripturae uocem pro parte Donati » (1. 845), il a noté " Hinc contra schisma donatistarum » (LYON 788, f. 72r, dans LYON 603). Puis vient la note déjà citée : à la hauteur de «In canticis, inquiunt, canticorum loquitur sponsa » (1. 882), il a écrit « Propositio stulta donatistarum, ex cantico canticorum » (LYON 788, f. 72v, dans LYON 603). Après la dispute sur le Cantique, à la hauteur de «Sed dic aliud quod te dicebas esse dicturum » (1. 1025), on lit de sa main «Item alia, ex abacuc propheta» (LYON 788, f. 74v, dans LYON 603). Et enfin en face de «Dicunt et aliud : Cyrineus, inquit, quidam Simon» (1. 1092), sa note annonce : «Item alia ex euangelio » (LYON 603, f. 1v). Le Florus lecteur du sermon 46 d'Augustin est donc parfaitement conscient du contexte anti-donatiste et de la progression de la réflexion augustinienne, et il s'y montre particulièrement attentif.
} 
Pierre Chambert-Protat, « Les centons augustiniens de Florus de Lyon : minutie, érudition et vulgarisation », dans Revue d'études augustiniennes et patristiques 60/2 (2014), p. 349-379 <halshs-01459687>

Ce faisant, Florus parvient pourtant à constituer un texte tout-à-fait équilibré. En guise d'introduction, on lit d'abord (passage a) une brève exhortation à être attentif, adressée au fidèle de bonne volonté (chez Augustin, c'était une apostrophe ad hominem du Donatiste imaginaire). Puis vient un lemme $(b-d)$ : une citation intégrale du dialogue des époux (qu'Augustin ne cite jamais que par petits bouts). L'épithalame se prolonge dans le dialogue de l'Église et du Christ $(\mathrm{d}-\mathrm{h})$. Puis paraît la question de l'appartenance à l'Église $(\mathrm{h}-\mathrm{i})$; et enfin la sentence augustinienne sur la saine interprétation du midi (j). Ce travail est le fait d'un homme véritablement habile, puisque pas un iota n'est introduit, pas un iota n'est altéré, et l'ordre du texte originel est parfaitement respecté. Pour parvenir à ce résultat, il ne suffisait pas de prendre des phrases entières çà et là pour les juxtaposer : comme le montre bien l'emplacement des « coutures » entre les passages, il fallait s'arrêter et reprendre toujours en des endroits précis dont la jonction ne nécessiterait aucune adaptation et ne trahirait aucune distorsion, fût-ce au beau milieu d'une phrase, («ubi cubas | in meridie », passages b/c).

Enfin, le style du centon lui-même est tout différent du texte original : bien loin de l'oralité si spontanée du sermon d'Augustin, bien loin de l'ambiance de prétoire qu'il a su y susciter en convoquant les Donatistes, le centon de Florus est calme et méthodique, détaché et tout spirituel. En définitive, le centon ainsi constitué est complètement différent d'Augustin — mais son texte est intégralement d'Augustin. En débarrassant l'Augustin historique de toute la fièvre de la polémique anti-donatiste, Florus semble vouloir révéler l'Enseignement d'un père spirituel tout empreint de grauitas, un Augustin Père de l'Église pour les temps carolingiens.

\section{Le centon Ex libro V de ciuitate Dei sur le destin}

Cette analyse trouve un écho dans d'autres centons augustiniens de Florus, à commencer par le centon Ex libro $V$ de ciuitate Dei, que Florus a réutilisé ${ }^{20}$ dans son violent traité contre le De praedestinatione de Jean Scot.

Au début du livre cinq de La Cité de Dieu, Augustin se demande "pourquoi Dieu a fait à l'Empire romain païen la grâce d'une telle grandeur et longévité », | car «la cause de cette grandeur ne saurait être ni fortuite ni fatale $»^{21}$. Le fortuit ne le retient pas, mais il accorde un

\footnotetext{
${ }^{20}$ On lira ce centon dans son édition princeps et unique, celle de Klaus ZECHIEL-ECKES, "AugustinusRezeption... », p. 51-56. Remplois dans le traité Aduersus Iobannem : PL 119, 142D-144C.

${ }^{21}$ Nous suivons l'édition de B. DOMBART et A. KALB dans le volume CCSL 47, p. 128 sqq. : «Uideamus qua causa Deus (...) Romanum imperium tam magnum tamque diuturnum esse uoluerit » (praefatio 1. 5.7-8); «Causa ergo magnitudinis imperii Romani nec fortuita est nec fatalis » (cap. i 1. 1-2).
} 
Pierre ChAmbert-Protat, « Les centons augustiniens de Florus de Lyon : minutie, érudition et vulgarisation ", dans Revue d'études augustiniennes et patristiques 60/2 (2014), p. 349-379 <halshs-01459687>

long examen (cap. i-xi) à la question du fatal : le destin, fatum. Il commence (cap. i-vii) par contrecarrer méthodiquement l'astrologie, Posidonius et les diverses façons de lier les actes humains à la position des astres. Il examine ensuite (cap. viii-x) le « destin» au sens de ce que nous appellerions "nécessité » : le destin comme enchaînement nécessaire de toutes les causes («omnium conexio seriesque causarum», cap. viii 1. 2-3; «ipse causarum ordo et quaedam conexio », cap. viii 1. 5-6). Il se fait alors l'adversaire de Cicéron : celui-ci, ayant posé comme prémisse que liberté humaine et prescience divine s'excluent mutuellement, a délibérément préféré à la seconde la première, sans laquelle aucune vie sociale n'est possible. Or, le chrétien ne peut exclure ni la liberté humaine, ni la prescience divine : il lui faut absolument conserver les deux. L'enjeu pour Augustin est alors d'invalider la prémisse du raisonnement, en montrant pourquoi il est faux de dire qu'elles s'exclueraient mutuellement.

C'est dans ces trois chapitres que Florus cisèle son centon. Le fait lui-même est remarquable : la question de la prédestination n'est pas la question du destin. La question de la prédestination est celle de savoir si et comment Dieu a décidé, pour chaque âme, de ce qu'il adviendra d'elle après la mort — ruine ou salut. Dans la mesure où saint Paul a déjà affirmé qu'il l’a décidé pour les saints, la question se pose pour les autres : le fait qu'ils ne sont pas saints correspond-il, en Dieu, à un acte positif de décision, acte symétrique de celui par lequel il a élu les élus ? Ou bien le défaut d'élection correspond-il à un défaut de décision ${ }^{22}$ de la part de Dieu ? Pour les théologiens carolingiens, là est la question. Au fil de leur réflexion, comme il faut aussi que le jugement ne soit pas un pur arbitraire et donc que Dieu juge avec justice, ils en viennent à s'interroger sur la valeur devant Dieu (le mérite) des actes opérés avant la mort : et comme devant un tribunal humain, cette valeur est proportionnée, ou du moins conditionnée, à la liberté, qui doit avoir présidé à l'acte. C'est alors seulement qu'on rejoint la question - non théologique mais philosophique - agitée ici par Augustin : le déterminisme, la mise en concurrence de la nécessité et de la liberté. Ces trois chapitres d'Augustin ont pour objet exclusif cette question philosophique qui concerne les vivants, et à aucun moment il n'évoque la question de la prédestination touchant le devenir post mortem. C'est bien Florus lui-même qui, ayant saisi les profondes implications philosophiques de la question théologique (à la faveur sans doute de la querelle carolingienne sur la prédestination), vient chercher l'autorité du Père de l'Église jusque dans la question philosophique.

\footnotetext{
${ }^{22}$ Le «défaut de décision » lui-même serait, de toute façon, un acte délibéré de la part de Dieu. Aucun théologien n'explique la damnation par un Dieu indécis.
} 
Pierre Chambert-Protat, « Les centons augustiniens de Florus de Lyon : minutie, érudition et vulgarisation », dans Revue d'études augustiniennes et patristiques 60/2 (2014), p. 349-379 <halshs-01459687>

| Méthode et intention du centon en regard de sa source

Quant à la facture du centon, elle n'est pas sans rapport avec le centon sur le Cantique. Remarquons d'abord l'identité matérielle de la méthode : certains passages sont clos ou ouverts au milieu d'une phrase, mais de telle façon que la couture soit invisible dans la copie au propre (« ut | auferat »; «Ipse itaque | ex his »); - les douze passages retenus suivent dans le centon le même ordre que dans le texte original ; - pas un iota n'est ajouté, pas un n'est modifié ${ }^{23}$.

Le choix des passages à retenir ou à écarter est également guidé par une intention similaire au centon sur le Cantique. Florus supprime d'abord les allusions à «ceux qui » tiennent tel ou tel discours, si bien que la troisième personne du pluriel tribuunt (cap. viii 1. 7-8), qui chez Augustin a ces gens-là pour sujet, prend chez Florus un sens impersonnel. Il écarte les vers de Sénèque et ceux d'Homère, avec leur commentaire (cap. viii 1. 12-35). Chez Augustin, Cicéron n'intervient qu'à ce point du texte : par son découpage, Florus le fait apparaître presque dès le début du centon, faisant de lui le principal objet de la réflexion. De Cicéron et de la présentation qu'en fait Augustin, Florus ne retient que l'essentiel : ainsi dans la phrase «Hos Cicero ita redarguere nititur, ut non existimet aliquid se aduersus eos ualere, nisi auferat diuinationem » (cap. ix 1. 12), il écarte la négative pour ne conserver que l'enseignement positif de la phrase : « ut | auferat diuinationem ». Il conserve le fait que Cicéron nie la prescience et les prophéties («et Dei praescientiam negat et omnem prophetiam ", cap. ix 1. 5-6), il conserve la condamnation de ce fait («negare praescium futurorum apertissima insania est », cap. ix 1. 13-14), mais il délaisse entre les deux toutes les précisions sur les textes et la démarche cicéroniens : «opponendo sibi quaedam oracula... » (cap. ix 1. 7-8), «Cottam fecit disputantem de hac re aduersus Stoicos in libris de deorum natura...» (cap. ix 1. 17-19). Les Stoïciens justement, qui chez Augustin se disputent la corde avec Cicéron, sont délibérément expulsés du centon, comme on le voit bien au passage suivant : Florus a gardé « quod Cicero timuit, ut oppugnaret praescientiam » et écarté la fin de la phrase : « et Stoici, ut non omnia... » (cap. ix 1. 32-33).

Ne prolongeons pas ce catalogue de faits : au terme du travail de Florus, dont ces détails donnent un aperçu éloquent, nous obtenons un centon très abstrait, bien moins complexe que le texte d'Augustin, dépouillé de digressions, débarrassé de tout ce qui nécessiterait des éclaircissements d'histoire littéraire, expurgé de toutes les considérations philosophiques ne concernant pas directement la question centrale du fatum (les différentes natures des causes et des

\footnotetext{
${ }^{23}$ Là encore, les préparations de Florus sont conservées dans le manuscrit de la Cité de Dieu LYON, B.M., 607, ff. $112 \mathrm{v}-113 \mathrm{v}$; elles sont par conséquent consultables en très haute définition sur http://florus.bmlyon.fr/visualisation.php? cote $=$ MS0607\&folio $=112 \mathrm{~V}$.
} 
Pierre Chambert-Protat, « Les centons augustiniens de Florus de Lyon : minutie, érudition et vulgarisation ", dans Revue d'études augustiniennes et patristiques 60/2 (2014), p. 349-379 <halshs-01459687>

volontés, cap. ix 1. 103-121 ; la soumission des volontés, cap. ix 1. 133-138 ; cap. ix 1.162 - cap. $x$ 1. 6). Surtout, à partir d'un texte où Augustin rencontre et explore la richesse de la philosophie et de la littérature païennes, nous obtenons chez Florus le face-à-face | d'un sage Augustin et d'un Cicéron non seulement païen, impie et sacrilège, mais même bête et méchant - à cet égard, on relèvera la maligne suppression des mots : [ipse Cicero] «ut uir magnus et doctus et uitae humanae plurimum ac peritissime consulens » (cap. ix 1. 56-57).

Le centon Ex libro $V$ de ciuitate Dei ne trahit pas l'opinion d'Augustin, en ce sens qu'il ne fait pas dire à Augustin le contraire de ce qu'il voulait dire en dernier lieu : le centon restitue bel et bien le fatum tel qu'Augustin entendait l'expliquer dans ces trois chapitres, à travers la contradiction des philosophes. Mais il saute directement de conclusion en conclusion, sans s'embarrasser de tout ce qui étaye ou promeut le raisonnement : la parole, l'arrêt d'Augustin suffit. Florus n'est donc pas à proprement parler malhonnête vis-à-vis de la pensée d'Augustin ; mais dans ce centon particulier, sa méthode fait une victime. Le Cicéron du centon florien, en regard du Cicéron de l'original augustinien, peut bien faire parler d'un Florus malhonnête. Mais après tout, est-il étonnant que le philosophe païen ne bénéficie pas du même a priori que le Père de l'Église, dans l'œil du théologien carolingien ?

\section{Un commentaire à deux voix du Notre Père}

D'une certaine façon, c'est le cas de figure inverse qui se présente dans un troisième centon augustinien. Celui-ci n'est pas un texte isolé, comme l'Ex libro $V$ de ciuitate Dei, mais un item dans une compilation.

Passage, extrait, item, centon, compilation... Pour une typologie de la citation patristique chez Florus.

Alors que le centon se présente comme un texte, la compilation se présente comme une série de textes. Ceux-ci, courts ou longs, sont autant d'items distincts. La compilation est à bon droit regardée comme une œuvre, en ce que les items qui la composent forment une série cohérente et ordonnée par un projet déterminé, parfois manifestée par une numérotation. Mais les items y sont clairement distincts, aussi bien dans leur présentation matérielle - ils sont séparés par une rubrique, marqués par un numéro d'ordre, une lettrine, un pied de mouche ou un retour de paragraphe en leur début - que dans leur composition littéraire, puisque sans lien grammatical ou syntaxique entre eux. Ainsi Florus compilateur s'évertue-t-il à isoler les items en supprimant soigneusement tous les ergo, itaque, enim, uero, autem, igitur, etc. de leur première phrase - 
Pierre Chambert-Protat, « Les centons augustiniens de Florus de Lyon : minutie, érudition et vulgarisation », dans Revue d'études augustiniennes et patristiques 60/2 (2014), p. 349-379 <halshs-01459687>

même lorsqu'il sépare en deux items successifs un texte pourtant continu dans sa source ${ }^{24}$. En revanche, auteur de $\mid$ centon, Florus fait exactement l'inverse en prenant bien soin de masquer toutes les coutures - quitte cette fois, nous l'avons vu, à garder un itaque en fin de passage, en prévision de la phrase qui se formera là avec le passage suivant...

À l'œil nu, les items de la compilation se présentent tous de la même façon : c'est le philologue qui, dans un second temps, en comparant systématiquement chaque item avec sa source, détermine que tel extrait correspond à un passage, tel autre extrait à plusieurs passages concaténés. À cet égard, on peut distinguer dans les compilations de Florus de Lyon trois types d'items : des items simples, "tuniques sans couture » dont le texte est tiré continûment de la source (un extrait $=$ un passage ${ }^{25}$; des items composites, où sont concaténés plusieurs passages (un extrait = de deux à cinq passages) ; et des items centons, produits d'un travail de découpage beaucoup plus élaboré et concaténant, par conséquent, un nombre bien plus important de passages (un extrait $=$ une dizaine de passages au moins). Bien entendu, plus le niveau de complexité est élevé, et plus la fréquence de ces objets diminue. Ainsi, dans la compilation des $B$. Augustini sententiae de praedestinatione qui compte cent vingt-neuf items, on recense cent-dix neuf items simples et neuf composites (items $n^{\circ} 10,17,38,54,58$ : deux passages ; ${ }^{\circ} 11$ et 96 : trois passages; $\mathrm{n}^{\circ} 110$ et 125 : cinq passages) contre un seul centon (item $\mathrm{n}^{\circ} 114$ : douze passages). C'est ce dernier que nous voudrions examiner.

\section{La compilation en général et l'item en particulier}

La compilation des B. Augustini sententiae de praedestinatione nous est conservée par un unique manuscrit, le codex GENT, U.B., 249 (celui-là même qui nous transmet également le centon Ex

\footnotetext{
${ }^{24}$ Le cas n'est pas rare. Ainsi, dans les B. Augustini sententiae de praedestinatione, les extraits numéro 15-16, 2122, 24-25 et 25-26, 34-35, 39-40, 69-70, 77-78, 80-81, 82-83, 86-87, 94-95, 102-103, 105-106, 111-112, 124125 : chaque fois, en découpant deux extraits distincts dans un passage continu d'Augustin, Florus supprime le mot de liaison de la première phrase du second extrait. Sur notre distinction entre « extrait » et «passage », cf. plus haut note 13 .

${ }^{25}$ On fera abstraction des suppressions d'un seul mot en début d'extrait (ergo, itaque, enim, igitur...), sans quoi il faudrait regarder presque tous les items comme composites. Symétriquement, lorsque Florus intercale un inquit dans la première phrase de l'extrait, on n'en déduit évidemment pas qu'il y a là deux citations patristiques distinctes, quoique matériellement le texte patristique soit interrompu. Aussi bien ces deux opérations, souvent liées (Florus place souvent son inquit à l'emplacement qu'occupait le mot de liaison dans la phrase originale, comme un dernier scrupule de minutie), ne sont pas la même chose que de retirer une phrase ou un membre de phrase porteur d'un sens propre et positif, fût-il court : cas qui justifie qu'on parle d'un item composite.
} 
Pierre Chambert-PRotat, «Les centons augustiniens de Florus de Lyon : minutie, érudition et vulgarisation », dans Revue d'études augustiniennes et patristiques 60/2 (2014), p. 349-379 <halshs-01459687>

libro $V$ de ciuitate Dei). Ce manuscrit est probablement l'apographe d'un dossier personnel de Florus $^{26}$. Cet original de la compilation est perdu, mais nous avons conservé dans le manuscrit LYON, B.M., 608 la principale source des extraits de la compilation, soigneusement préparés de la main de Florus ${ }^{27}$.

| L'item 114 de la compilation (Ex libro de praedestinatione sanctorum itemque de dono perseuerantiae ad Prosperum et Hilarium VII) concatène, dans l'ordre du texte source, douze passages du De dono perseuerantiae ${ }^{28}$ (2,3-5,9 : BA 24, pp. 606-618) prélevés par Florus sur son exemplaire, le manuscrit LYON, B.M., 608, ff. 135v-138r. Nous donnons ici en annexe le texte de ce centon, repris du manuscrit de Gand, et collationné sur les préparations de Florus conservées dans le manuscrit de Lyon.

\section{Méthode et intention du centon en regard de sa source}

Le centon voulu par Florus constitue un commentaire ad litteram du Notre Père. Certes, ce passage d'Augustin tout entier en est un : à ce titre, le centon de Florus représente essentiellement un abrégé de celui d'Augustin. Mais quel abrégé ? Les choix de Florus révèlent deux projets.

Le premier projet est similaire à celui que nous avons mis au jour dans les deux centons étudiés plus haut : il s'agit de composer un commentaire purement spirituel à partir d'un réquisitoire anti-pélagien. Les Pélagiens et la lutte contre les Pélagiens traversent et promeuvent le texte d'Augustin : "contra ea quae futura erant Pelagianorum uenena... nam tria sunt, ut scitis, quae maxime aduersus eos catholica defendit Ecclesia » $(608,1-4)$; « et caetera quae contra

\footnotetext{
${ }^{26}$ Nous espérons l'avoir montré à l'occasion du récent colloque « La querelle carolingienne sur la prédestination » (cf. plus haut note 3).

${ }^{27}$ Ce manuscrit est intégralement consultable en ligne, cf. plus haut note 8. Huit extraits ne viennent pas de ce manuscrit : un extrait du concile d'Orange (A.D. 529) qui mit fin à la querelle gauloise sur la prédestination, et sept extraits de l'In Iohannem tract. 53 d'Augustin. Proviennent du manuscrit LYON, B.M., 608 les cent vingt-et-un extraits des De perfectione iustitiae hominis, De natura et gratia, Epistulae 214 et 215 et de gratia et libero arbitrio, De correptione et gratia, De praedestinatione sanctorum et de dono perseuerantiae. Sur cette compilation et sur le centon Ex libro $V$ de ciuitate Dei, lui-même préparé sur le manuscrit LYON, B.M., 607, voir K. ZECHIEL-ECKES, "Augustinus-Rezeption... ».

${ }^{28}$ Pour les références des passages que nous citons, nous donnons la pagination et délinéation de la Bibliothèque Augustinienne : Euvres de saint Augustin, 24. Troisième série : La grâce. Aux moines d'Adrumète et de Provence (De gratia et libero arbitrio, De correptione et gratia, De praedestinatione sanctorum, De dono perseuerantiae). Texte de l'édition des Mauristes. Introduction, traduction et notes par Jean CHÉNÉ et Jacques PINTARD, Paris, Desclée de Brouwer, 1962.
} 
Pierre Chambert-Protat, « Les centons augustiniens de Florus de Lyon : minutie, érudition et vulgarisation ", dans Revue d'études augustiniennes et patristiques 60/2 (2014), p. 349-379 <halshs-01459687>

Pelagianos dona Dei esse defendimus... »(610,14-15) ; «hoc enim Pelagiani audent dicere... » (616,17-18) - mais ils ne se trouvent plus nulle part dans le centon de Florus. L'oblitération de la polémique anti-pélagienne correspond, dans ce centon, à l'oblitération de la polémique antidonatiste dans le centon Etenim illa cantica, et de la polémique anti-païenne dans le centon Ex libro $V$ de ciuitate Dei.

Le second projet est plus singulier, et propre à la source où Florus vient puiser. À travers tout le passage, Augustin s'appuie en fait sur le De oratione dominica de Cyprien de Carthage, qu'il cite à quatre reprises. Le fait est indécelable dans le centon, où le nom de Cyprien n'apparaît même pas. Et pourtant, Florus a détouré soigneusement ces quatre citations, pour leur confier dans son centon le commentaire des première (passages $b-c)$, quatrième (i) et cinquième $(k)$ demandes du Notre Père : au sujet de ces trois demandes-là, Florus ne retient rien de ce qu'Augustin dit de son cru. Quant aux deuxième, troisième et sixième demandes, comme Augustin ne citait pas Cyprien à leur propos, c'est alors le commentaire personnel d'Augustin que Florus a repris $(\mathrm{d}, \mathrm{e}-\mathrm{g}, \mathrm{l})$.

| Au détriment d'Augustin, donner la parole à Cyprien autant que possible, mais sans jamais le mentionner : l'intention apparaît très clairement à l'étude des découpages et coutures. Ainsi Florus écarte-t-il cette phrase qui suit immédiatement le passage a : «legite aliquanto intentius eius [scil. orationis dominicae] expositionem in beati Cypriani martyris libro, quem de hac re condidit, cuius est titulus : De dominica oratione... » (608,23-25). Au début du passage b, le texte d'Augustin : "sic loquitur: "dicimus, inquit, Sanctificetur...” " devient chez Florus : «Dicimus enim Sanctificetur... » Par la suite, Augustin saute un passage de Cyprien avant de le citer de nouveau : «Et paulo post de hac ipse re adhuc disputans, et docens nos (...) "Haec, inquit, sanctificatio...” - Florus, lui, fait comme s'il s'agissait de la suite du texte, et transcrit sans interruption : «Haec itaque sanctificatio... » Même manège entre les passages h et i, où Florus écarte cette phrase d'Augustin : «Ubi beatus Cyprianus ostendit quomodo et hic intelligatur perseuerantia postulari. Ait quippe inter caetera...»(614,17-19). Puis derechef pour le passage $\mathrm{k}$ : Florus élimine toutes les allusions au fait qu'il s'agit d'une citation, soit avant elle : «attendite quomodo istos [Pelagianos] gloriosissimus Cyprianus interimat. Cum enim locum ipsum dominicae orationis exponeret, ait inter caetera... »(616,25-27), soit après elle : " et caetera quae hic inserere longum est » $(618,7)$.

Le centon, une fois copié au propre, se présente à son lecteur comme un commentaire d'Augustin sur le Notre Père, et seule la confrontation ligne à ligne avec le texte original révèle qu'il s'agit en réalité d'un commentaire à deux voix de Cyprien et d'Augustin. Ce choix de Florus 
Pierre Chambert-PRotat, «Les centons augustiniens de Florus de Lyon : minutie, érudition et vulgarisation », dans Revue d'études augustiniennes et patristiques 60/2 (2014), p. 349-379 <halshs-01459687>

reflète l'ambivalence de son travail. Il ne pouvait qu'être intéressé de trouver des citations de Cyprien chez Augustin : d'ailleurs, quand on examine son exemplaire de travail, on remarque à la hauteur de la phrase "legite aliquanto » une note de sa main, érasée mais lisible grâce aux numérisations en très haute définition mises en ligne par la Bibliothèque municipale de Lyon : "NOTA de libro beati cypriani, de dominica oratione " (LYON, B.M., 608, f. 135v, 1. 24, in marg.). L'érudit Florus a donc voulu conserver soigneusement ces citations ${ }^{29}$; mais Florus le vulgarisateur ne veut pas égarer le simple fidèle avec des considérations d'histoire littéraire, et il écarte soigneusement tout ce qui pourrait réclamer une «note de bas de page ». Ce qui semble importer au théologien qui se met ici au service du fidèle, c'est donc avant tout l'Autorité en tant que telle : pour Florus, qui connaît la source, l'autorité est en quelque sorte | «double » dans ces lieux où Augustin sanctionne Cyprien - pour le lecteur de son centon, l'autorité d'Augustin est suffisante.

${ }^{29}$ Dans le manuscrit PARIS, B.n.F., lat. 10592, antiquissimus des œuvres de Cyprien sur lequel Florus a travaillé, le troisième de nos quatre passages du De dominica oratione apparaît entre crochets, corrigé et ponctué à la manière caractéristique de Florus. Mais le passage ainsi prélevé et préparé est plus ample que le passage cité par Augustin et repris dans notre centon : il commence plus haut à «Panis uitae Christus est » et finit plus bas à «et corpore non recedamus » (f. 43rb,13-vb,21 : CYPR. domin. orat., 18 : CCSL 3A, 101,327-102,347). Ce travail de préparation n'a rien à voir avec le centon; en revanche, il correspond exactement à une citation utilisée par Florus dans son plaidoyer contre Amalaire au concile de Quierzy en 838 (MGH Conc. 2,2, 773,21-33). 
Pierre ChAmbert-Protat, « Les centons augustiniens de Florus de Lyon : minutie, érudition et vulgarisation », dans Revue d'études augustiniennes et patristiques 60/2 (2014), p. 349-379 <halshs-01459687>

\section{Florus auteur de centons : l'érudit et la foi des simples}

Dans ces trois centons donc, non seulement la méthode ${ }^{30}$, mais encore l'intention reste d'une certaine façon la même : il s'agit de dégager l'enseignement spirituel d'Augustin de tous les détails d'ordre contextuel et historique ${ }^{31}$. $\mid$ Et pourtant, nous connaissons par ailleurs un Florus très friand, à titre personnel, de pareils détails.

\footnotetext{
${ }^{30}$ Sans nous étendre outre mesure, nous attirons l'attention sur un contre-exemple apparent : un item de la grande Compilation augustinienne sur l'Apôtre, FLOR. LvGD. expos. Aug. in Gal. exc. 88 (CCCM 220B, pp. 264267). Cet item concatène douze passages tirés du livre XV de La Cité de Dieu, mais la méthode, une fois n'est pas coutume, se démarque nettement de ce que nous avons décrit pour nos trois centons. Le texte augustinien où Florus vient puiser ici est une longue méditation de Gal. 4, 21-31, dont le texte est d'ailleurs cité in extenso par Augustin un peu après avoir commencé son commentaire. Dans sa compilation augustinienne, arrivé à Gal. 4, 22-24, Florus saisit l'opportunité : il s'approprie l'idée d'un long commentaire suivi des dix versets. Ce long item une fois conclu sur le commentaire de Gal. 4, 31, il reprend le fil de sa compilation où il l'avait laissé, c'est-à-dire à Gal. 4, 22-24, dont il reproduit le lemme pour le faire commenter d'un nouvel item ex tractatu psalmi CXVIII (FLOR. LvGD. expos. Aug. in Gal. exc. 89 : CCCM 220B, pp. 267-268). En somme, Florus a trouvé intéressant d'avoir un commentaire plus suivi cette fois qu'à l'accoutumée, et a donc voulu le reproduire sans en disperser les morceaux en divers endroits de sa compilation; mais il a tout de même retravaillé le texte. Augustin produisait un long lemme de onze versets, autour duquel gravitaient les commentaires de chaque phrase, mais dans un ordre qui ne correspond pas à l'ordre paulinien. Florus, lui, citera Paul phrase par phrase, allant chercher pour chacune ses commentaires augustiniens où ils se trouvent (c'est-à-dire sans respecter l'ordre du texte de La Cité de Dieu). Pour donner une idée synthétique de cette « remise en ordre » opportuniste des douze passages, prenons-les tels qu'ils se présentent dans l'item de Florus, et numérotons-les de (a) à (l). On obtient la séquence suivante : [lemme 1] a-b-[lemme 2]-c-d-e-[lemme 3]-f-g$\mathrm{h}-[$ lemme 4]-i-[lemme 5]-j-k-[lemme 6]-1. Si maintenant nous les remettons dans l'ordre du texte original d'Augustin, en signalant les continuités par un trait d'union et les discontinuités (passages sautés) par un pointvirgule, la séquence devient : f-e-[lemme $1-2-3-4-5-6]-\mathrm{c}-\mathrm{k}-\mathrm{d}-\mathrm{l}-\mathrm{a}-\mathrm{g}-\mathrm{h}-\mathrm{b} ; \mathrm{i} ; \mathrm{j}$. On aurait pu croire à une dispersion complète : en réalité, la comparaison avec le texte original montre que l'item de Florus reproduit toute la méditation d'Augustin (CCSL 48, 454,1,56-456,3,25), sans en rien omettre - et au contraire, en lui adjoignant deux passages complémentaires du livre XVI (les passages i et j). Les découpages et les coutures, cependant, sont loin de la finesse observée dans les centons, si bien qu'on devrait voir là plutôt un « item composite » d'une envergure exceptionnelle qu'un centon : Florus n'a changé que l'ordre des passages augustiniens, pour leur faire suivre l'ordre du texte paulinien cité phrase à phrase. Et en cela, cet item de la Compilation augustinienne sur l'Apôtre est une parfaite image en réduction de la Compilation augustinienne sur l'Apôtre.

${ }^{31}$ Cela jette, à défaut de lumière, une sorte de clair-obscur sur un fragment patristique de Florus dont la source est perdue : l'Ex libris Tertulliani de execrandis gentium diis (Cf. Anne-Marie TURCAN-VERKERK et Marie TURCAN, «Faut-il rendre à Tertullien l'Ex libris Tertulliani de execrandis gentium diis du manuscrit Vatican latin 3852 ? » dans
} 
Pierre Chambert-Protat, « Les centons augustiniens de Florus de Lyon : minutie, érudition et vulgarisation », dans Revue d'études augustiniennes et patristiques 60/2 (2014), p. 349-379 <halshs-01459687>

\section{Le travail d'un authentique érudit}

Nous le voyons oblitérer complètement dans ses centons les questions de contexte polémique, lui qu'on présente si souvent comme un pourfendeur d'hérésie particulièrement acharné. Nous avons vu qu'il supprime les Donatistes du centon Etenim illa cantica - et pourtant le même Florus a noté au même endroit dans la marge de son manuscrit : "Propositio stulta donatistarum ${ }^{32}$.

Bien plus, dans le centon sur le Notre Père, Florus supprime les précisions apportées par Augustin sur sa source ( in beati Cypriani martyris libro, quem de hac re condidit, cuius est titulus : De dominica oratione », 608,23-25) ; même phénomène, nous l'avons signalé, pour les textes cicéroniens décrits par Augustin dans le texte où Florus a découpé son centon Ex libro $V$ de ciuitate Dei. Et pourtant c'est justement ce type de précisions «bibliographiques» que Florus s'attache à mentionner quasi systématiquement : soit dans ses titres (le titre du centon ex libro $V$ de ciuitate Dei n'est qu'un exemple parmi d'autres), soit dans ses opuscules, lorsqu'il s'apprête à citer quelqu'un ${ }^{33}$. Corollaire de cette discipline personnelle, ne pas avoir signalé un long emprunt à l'Hypomnesticon ${ }^{34}$ vaut à Hraban Maur quelques remarques acides.

REAug 46 (2000), pp. 205-271). Notre analyse du Florus auteur de centon parle, croyons-nous, en faveur de la paternité florienne : tout indique qu'il s'agit bien d'un centon de son cru. Dès lors, la comparaison avec les centons augustiniens peut rassurer : le texte du centon est à la lettre le texte de Tertullien tel que Florus le connaissait, et Marie Turcan l'a bien montré en cherchant à déterminer l'emplacement des « coutures» du centon. Mais notre étude peut aussi décourager : car sans possibilité de comparer avec le texte original, il parait irrémédiablement impossible de savoir avec certitude où se trouvent ces « coutures». Ce centon nous conserve des fragments d'un ouvrage perdu de Tertullien, mais nous ne pouvons savoir précisément combien de fragments, ni où commence et où finit chacun d'eux.

${ }^{32}$ Cf. plus haut notre note 19.

${ }^{33}$ Pour ne donner que l'exemple de son Absolutio cuiusdam quaestionis: "beatus Ambrosius in expositione epistolae ad Ephesios de eadem re sic dicit... » (PL 121, 1073,18-19) ; «in libro de uiris inlustribus beatus testatur Hieronymus... » (ibid., 1073,30-31) ; «in quadam itaque epistola ad Optatum episcopum ita loquitur [Augustinus] ... » (ibid., 1075,27-29); «item in libris de ciuitate Dei...» (ibid., 1076,57); «iam uero in libris de baptismo paruulorum... » (ibid., 1078,4) ; « ex libro etiam eiusdem beati doctoris, qui usitatius Enchiridium appellatur... » (ibid., 1081,48-49).

${ }^{34}$ Dans le De tribus epistolis : «Tacito nomine libelli Hypomnesticon subiungit et adiungit eius uerba, et sensus uelut uno tenore et ordine sermonis sui » (PL 121, 1061,55-57) ; et après avoir reproduit le passage incriminé : «Haec igitur omnia quia, ut diximus, non sunt uerba eius sed manifeste ex iam dicto libello sumpta agnoscuntur... » (ibid., 1063,5-7). La troisième lettre à laquelle répond Florus dans son De tribus epistolis est en réalité l'opuscule de Hraban Maur De praedestinatione, avec une lettre-préface à Noting de Vérone (Epistola V ad Notingum cum libro de 
Pierre ChAmbert-Protat, « Les centons augustiniens de Florus de Lyon : minutie, érudition et vulgarisation ", dans Revue d'études augustiniennes et patristiques 60/2 (2014), p. 349-379 <halshs-01459687>

Sans même compter son habitude de porter des renvois aux livres bibliques, ses autres annotations marginales nous montrent partout un Florus doué d'une curio|sité aussi encyclopédique qu'érudite. Ainsi dans son exemplaire du sermon 46 d'Augustin : un peu avant le passage de notre centon, il a noté : «NOTA interrogationem ostiarii ecclesiae, more antiquo" (LYON, B.M., 788, f. 71r) ; puis plus bas : «NOTA item lybiam duobus modis dici » (LYON, B.M., 603, f. 1v). Les exemples abondent dans ses manuscrits de travail : nous avons déjà parlé de la note érasée par laquelle il renvoyait au De dominica oratione à la hauteur du passage d'Augustin où il a ciselé son commentaire « à deux voix » du Notre Père (LYON, B.M., 608, f. 135v). Dans le même ordre d'idée, en regard d'un passage où Augustin mentionne cet ouvrage (AvG. perseu. 8,20), il note : «NOTA librum beati ambrosii, de fuga saeculi »(LYON, B.M., 608, f. 142r). En regard d'un passage où Tertullien détaille le cas d'une femme qui a des visions durant les offices sacrés (TERT. anim. 9), il écrit : « NOTA formam ueteris celebrationis » (« codex Agobardinus» : PARIS, B.n.F., lat. 1622, f. 120v). Il relève une étymologie grecque dans la lettre 140 d'Augustin : «NotA græcam tristitiæ ethimologiam » (CitTÀ DEL VATICANO, B.A.V., Reg. lat. 331, f. 7v), et un passage sur le pélican chez Paulin de Nole (epist. 40) : «NoTA de pel[i]can[o] » (ibid., f. 47r). Dans la lettre 188, il relève une information sur la crise pélagienne : «NOTA cauendam pelagianam epistolam » (PARIS, B.n.F., n. acq. lat. 1443, f. 32v), et dans la lettre 190 une allusion à Tertullien : «NOTA de tertulliani somniis » (ibid., f. 107v). Dans la lettre 101, son attention est attirée par un passage sur les arts libéraux : «NOTA nimis quid de artibus liberalibus sentiendum sit. - POET' - ORAT' - PHYL' - HYS' - RYTHM' - MUS' » (ibid., f. 145v-146r). Et lorsque Eucher, s'adressant à Salonius, évoque le retrait au désert de ce dernier à l'âge de dix ans (EVCHER. instr. praef.), l'hagiographe ne peut que s'intéresser: "NoTA de sanctitate et institutione sancti salonii »(PARIS, B.n.F., lat. 9550, f. 37v). Dans ses Nota, Florus fait souvent l'effet d'un homme qui recenserait des entrées d'index, ou préparerait un article de dictionnaire.

Aussi, lorsque les circonstances l'exigent ou lorsque cela sert son argumentation, Florus se révèle capable de déployer dans sa prose une érudition tout-à-fait impressionnante. Pour ne prendre l'exemple que de ses traités sur la prédestination, ses explications par le grec sur les divergences entre versions latines de la Bible trahissent une connaissance experte du lexique, sans tomber dans une rigidité tout artificielle qui ferait correspondre strictement un unique mot grec à un unique mot latin ${ }^{35}$. Ses dissertations sur l'authenticité de l'Hypomnesticon du pseudo-

praedestinatione Dei, PL 112, 1530-1553 ; la lettre-préface a été éditée en 1899 par Ernst DÜMMLER : Ep. 22, MGH Epp. 5, p. 428). 
Pierre ChAmbert-Protat, « Les centons augustiniens de Florus de Lyon : minutie, érudition et vulgarisation », dans Revue d'études augustiniennes et patristiques 60/2 (2014), p. 349-379 <halshs-01459687>

Augustin, reposant sur des arguments de critique interne et externe, restent un modèle du genre $^{36}$. Sans doute, peu de gens en son temps pouvaient avoir une pareille conscience de toutes les questions que nous appelons «philologiques».

\section{| Une vulgarisation fondée sur une pédagogie bien comprise}

Florus l'érudit n'a aucune peine à faire preuve d'érudition. Dans les centons que nous avons étudiés, la méthode et le parti-pris inverses sont donc volontaires et maîtrisés. Ils correspondent à une volonté délibérée de ne pas noyer le lecteur sous des torrents de détails, mais de faire émerger pour lui ce que Florus regarde comme l'Enseignement d'Augustin ; de ne pas faire d'érudition, mais de la vulgarisation ; de ne pas tant nourrir la vie intellectuelle, que la vie spirituelle de son lecteur. Sans doute, il s'agit pour partie de faire le travail de secrétaire auquel Florus est accoutumé : assimiler l'immense matière de la bibliothèque afin d'en livrer la quintessence lorsque l'évêque pourra en avoir besoin. Mais il ne s'agit pas ici d'aller chercher les canons par lesquels l'évêque jugera péremptoirement une question épineuse, ce qui est encore un travail d'expert. Ici, Florus semble se mettre plutôt à la portée du fidèle ignorant, du simple clerc, qui a besoin d'explications spirituelles pour faire son salut, mais que l'érudition ne concerne pas ${ }^{37}$. Ce faisant, il se montre fidèle au projet formulé explicitement dans sa belle préface aux B. Augustini sententiae de praedestinatione : Florus propose dans ses centons une "substantificque moelle » du commentaire augustinien, "pour que le lecteur pieux et simple n'aille pas s'éreinter des grandes et nombreuses disputes de ce Père, ni carrément s'effrayer et se troubler de la profondeur et de la

\footnotetext{
${ }^{35}$ Par exemple dans l'Aduersus Iobannem (PL 119, 183D-184A ; ibid., 233D-234A) ou dans le De tenenda immobiliter (PL 121, 1096B-D). Eldrade de Novalèse avait commandé à Florus une révision du Psautier, pour laquelle Florus s'est livré à un véritable travail de collation. Il s'en explique dans une lettre qui mérite sa célébrité ; cf. P.-M. BogAERT, «Florus et le Psautier. La lettre à Eldrade de Novalèse », dans RBen 119 (2009), pp. $403-419$.

${ }^{36}$ Dans le De tribus epistolis (PL 121, 1044A-1047A ; ibid., 1051D-1054B) ou dans le De tenenda immobiliter (PL 121, 1106D-1110A). Interrogé également sur l'authenticité du De induratione cordis Pharaoni du pseudoJérôme, Florus refuse de se prononcer définitivement parce qu'il ne connaît pas le texte, mais il subodore la pseudépigraphie à partir des informations bio-bibliographiques dont il dispose sur l'œuvre de Jérôme, et donne quelques conseils sur les moyens de trancher la question à partir du texte. En d'autres termes, la critique externe le fait pencher en faveur de la pseudépigraphie, mais il refuse de trancher faute de pouvoir procéder lui-même à la critique interne.

${ }^{37}$ Nous pensons au public non érudit pour lequel s'inquiétaient, lors de la controverse sur la prédestination, Noting de Vérone et Hraban Maur, puis Hincmar de Reims dans sa lettre pastorale Ad simplices.
} 
Pierre ChAmbert-PRotaT, « Les centons augustiniens de Florus de Lyon : minutie, érudition et vulgarisation ", dans Revue d'études augustiniennes et patristiques 60/2 (2014), p. 349-379 <halshs-01459687>

difficulté de pareilles questions - mais qu'il apprenne, en un bref résumé, comment édifier fidèlement sa foi, et conformer sainement ses actes ${ }^{38}$. »

\section{Conclusion}

Plus généralement, nous voudrions avoir attiré l'attention sur cette habitude qu'avait Florus de Lyon, à côté de ses compilations bien connues, de découper ponctuellement des pages et des centons isolés. Les manuscrits médiévaux fourmillent de ce genre de petits textes, mal identifiés, souvent difficiles ou impossibles à rendre à qui que ce soit. Grâce à l'immensité sans exemple de la documentation manuscrite de première main qui nous reste de lui, Florus de Lyon nous apparaît | presque à son pupitre, le calame à la main : il nous offre une occasion rare d'observer « en train de se faire », étape par étape, une des activités de vulgarisation dont le moyen âge fut, par la suite, plus coutumier encore.

À cet égard, l'analyse de ses centons par la comparaison avec les textes où il puise et les manuscrits qu'il annote, est riche d'enseignements. La recherche sur Florus de Lyon a beaucoup révélé l'érudit, le quasi-philologue, et surtout le Marteau de l'Église de Lyon, prompt dans ses humeurs, catégorique dans ses jugements, brutal dans ses attaques, ne reculant pas devant l'insulte. Mais on ne peut négliger en lui le coureur de fond, le travailleur de l'ombre, ne disant jamais son nom, ne parlant jamais de lui-même que pour parler de sa mère Église, ne réclamant jamais rien pour lui, et soumettant l'étendue et la précision de sa science à l'impératif de la vie spirituelle, grâce à une fine intelligence pédagogique de la matière et des frères qu'il veut servir. C'est cet aspect, sans doute, qui a fait trouver en certaines de ses œuvres un sain aliment spirituel, longtemps après que son nom se fut effacé des mémoires.

Pierre CHAMBERT-PROTAT

LabEx HASTEC / SAPRAT EA 4116 / EPHE

\footnotetext{
38 «Ut deuotus et simplex lector, ne magnis et multiplicibus praedicti patris disputationibus fatigetur, uel etiam profunditate ac perplexitate tantarum quaestionum deterreatur aut perturbetur, breui compendio discat unde fidem suam fideliter aedificet, et actionem salubriter informet. » FLOR. LVGD., aug. sent., praefatio.
} 
Pierre Chambert-Protat, « Les centons augustiniens de Florus de Lyon : minutie, érudition et vulgarisation », dans Revue d'études augustiniennes et patristiques 6o/2 (20I4), p. 349-379 <halshs-0I459687>

\section{Ex b. Augustini libro de pastoribus (sermo xlvi) quaedam exposita de Cantico canticorum ( $(1,6-7)$}

L codex : Lyon, B.M., 603 : Ms. 788

$F$ main : Florus de Lyon

ed. édition : Augustinus, Sermo 46 de pastoribus d'après Augustin : Sermones de vetere testamento (I-50), éd. C. Lambot (Corpus Christianorum Series Latina 4I), Turnhout, 196I

Etenim illa cantica : enigmata sunt. Paucis intellegentibus nota sunt, paucis pulsantibus aperiuntur. Tene et deuote accipe aperta, ut merito tibi pandantur obscura. Quomodo eris penetrator obscurorum, contemptor manifestorum. ${ }^{\mathbf{b}}$ Etenim sic se habet textus ipse lectionis. Sponsa loquitur ad sponsum. Annuntia mibi quem dilexit anima mea, ubi pascis, ubi cubas ${ }^{c}$ in meridie. Ne forte fiam sicut operta, super greges sodalium tuorum. Hucusque uerba sponsae. Iam incipiunt uerba sponsi. ${ }^{\mathbf{d}} \mathrm{Nisi}$ cognoueris temetipsam o pulchra inter mulieres, exi tu in uestigiis gregum, et pasce hedos tuos in tabernaculis pastorum. Non in tabernaculo pastoris. Vide quomodo comminetur sponsus. Vide quemadmodum in periculo, quamuis ille dulcis, abstulit de medio blandimenta. Quam blande illa. 'Annuntia mihi quem dilexit anima mea, ubi pascis ubi cubas in meridie.' Venit enim medius dies quando ab umbrao cula concurrunt pastores, et 'forte' latebit me 'ubi tu pascis et ubi cubas'. Et uolo mihi annunties, 'ne forte fiam sicut operta', idest sicut occulta et non cognita. Ego enim manifesta sum. Sed 'ne sicut operta', sicut celata, incidam 'super greges sodalium tuorum'. Omnes enim heretici a Christo exierunt. Omnes qui facti sunt pastores mali, habentes 'greges' suos sub nomine Christi, illius 'sodales' fuerunt, illius conuiuium acceperunt. Sodales enim dicuntur, tamquam unius conuiuii. Latina lingua sic dicti sunt sodales, quasi simul edales, eo quod simul edant. Audi illum in psalmo arguentem sodales malos, idest unius conuiuii. Si inimicus inquit exprobrasset mibi, abscondissem me utique ab eo, et si super me magna locutus fuisset. Tu uero unanimis meus et notus meus, dux meus, qui simul mecum dulces capiebas cibos. Ergo multi sodales ingrati mensae dominicae, exierunt foras. Mali sodales, fecerunt sibi suas mensas, | erexerunt altaria contra altare. In eos ista timet errare. ${ }^{\mathrm{e}} \mathrm{Qui}$ sint tui, a te uolo audire. 'Annuntia mihi quem dilexit anima mea, ubi pascis, ubi cubas in meridie.' fTu mihi

4-5 Ct I,6 || 5-7 Ct I,7 || r6-18 Ps 54,13

I-3 loc. a ] Avg. serm. 46, xv, $35=$ CCSL 4I, 560,894-561,898 : praep. Lyon, B.M., 603 : Ms. 788, f. 72v,15-19. Proximam sententiam primo accepit $\boldsymbol{F}$ at reiecit in fine : Ecce tamen ut possumus fr(atre)s, haec uerba discutiamus; Aderit d(eu)s ut uideatis ibi [sibi a.c.] intellectum; (CCSL 4I, 561,899-900).

3-4 loc. b] Avg. serm. 46, xv, $36=$ CCSL 4I, 561,903-905: praep. Lyon, B.M., 603 : Ms. 788, f. 72v,22-24

4-5 loc. c] Avg. serm. 46, xv, 36 = CCSL 4I, 561,922-924 : praep. Lyon, B.M., 603 : Ms. 788, f. 73r,IO-II. Ultimam sententiam (hucusque - sponsi) primo reiecit $\boldsymbol{F}$ at accepit in fine.

5-19 loc. d] Avg. serm. 46, xv, $36=$ CCSL 4I, 561,927-561,949 : praep. Lyon, B.M., 603 : Ms. 788, f. 73r,13-73v,3

I9-20 loc. e] Avg. serm. 46, xv, $37=$ CCSL 4I, 563,962-964 : praep. Lyon, B.M., 603 : Ms. 788, f. 73v,I3-I5

20-22 loc. f] Avg. serm. 46, xv, $37=$ CCSL 4I, 563,966-968 : praep. Lyon, B.M., 603 : Ms. 788, f. 73v,16-I8

I enigmata] aenigmata $e d$. II 4 pascis ] pascit $a$. corr. L $\| 5$ operta] opera $a$. corr. L 5 sodalium ] solium $a$. corr. L $\| \mid 6$ gregum] grecum $a$. corr. L 6 pasce ] pascae $a$. corr. L 6 hedos] haedos $a$. corr. L hoedos ed. $\| 9$ meridie ] meridiae $a$. corr. L $\|$ I2 heretici] haeretici $e d$. \|I4 unius] unus $a$. corr. L $\|$ I5 edales] aedales $a$. corr. L I5 edant] aedant $a$. corr. L $\| \mathrm{I} 6$ inquit] om. a. corr. L $\| 17$ si $]$ om. a. corr. L I7 unanimis] unianimis ed. II 19 mensas ] menses $a$. corr. L 19 in eos ista timet errare ] in eo si statim et inerrare ut vid. a. corr. L $\| 20$ ubi pascis ] p. corr. L 
Pierre Chambert-Protat, « Les centons augustiniens de Florus de Lyon : minutie, érudition et vulgarisation », dans Revue d'études augustiniennes et patristiques 6o/2 (2014), p. 349-379 <halshs-0I459687>

dic quo eam : 'ne forte uelut operta' idest incognita, 'fiam super greges sodalium tuorum', incurram in greges hereticorum. ' 'Annuntia mihi'. Et ille qui commendat unitatem pastoris, ${ }^{\mathrm{h}}$ pastores autem reprobat qui multi esse uoluerunt, unitatem amiserunt, seuerissime non blande respondens, sed pro magnitudine periculi. 'Nisi cognoueris' inquit 'temetipsam o pulchra inter mulieres'. Pulchra es inter mulieres, sed agnosce te. Ubi te agnoscis? In toto orbe terrarum. Si enim pulchra unitas est in te, ubi diuisio foeditas est non pulchritudo. 'Nisi cognoueris temetipsam.' In me credidisti, agnosce te. In me quomodo credidisti? Quomodo et illi mali sodales consentiunt. Verbum carnem factum, natum ex uirgine, crucifixum, resurrexisse, ascendisse in caelum. In talem me credidisti, talem et illi sonant. Cognosce et te et me. Me in caelo. te toto orbe terrarum. Unum quemlibet ex ecclesia : tamquam ecclesiam Christus alloquitur. Nam quomodo ecclesia quaerit ecclesiam. ${ }^{\text {i Alloquitur }}$ unumquodque membrum ecclesiae suae Christus, tamquam suam ecclesiam. Et quid dicit. 'nisi cognoueris temetipsam o pulchra inter mulieres, exi.' Exire hereticorum est. Aut cognosce te, aut exi, quia 'si te non cognoueris' exitura es. Exitura quo ? 'In uestigiis gregum', sequendo malos greges. $\mathrm{Ne}$ forte putes quia oues sequeris si exis : audi quid sequitur. 'Exi tu in uestigiis gregum, et pasce hedos tuos' iam non oues. Nostis fratres ubi erunt 'hedi'. Ad sinistram erunt, omnes qui exierunt ab ecclesia. Manenti Petro, pasce oues meas. Exeunti haeretico : 'pasce hedos tuos'. j Et 'meridiem' intellego feruorem spiritalium, flagrantem igne caritatis, splendentem lumine ueritatis ? Nam dicitur in quodam psalmo. Dexteram tuam notam fac mibi, et eruditos corde in sapientia. 'Dexteram' non 'hedos', 'et eruditos corde in sapientia'. Ipsi sunt meridies. Vnde dicitur ad prophetam, et tenebrae tuae sicut meridies erunt.

\footnotetext{
36 Io $2 \mathrm{I}, \mathrm{I7} \quad$ || 38 Ps $89, \mathrm{I2} \quad$ || $39-40$ Is $58, \mathrm{IO}$

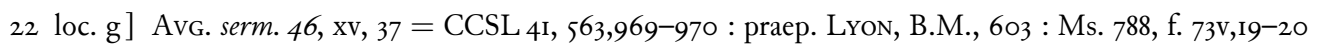
22-30 loc. h] Avg. serm. 46, xv, $37=$ CCSL 4I, 563,97I-564,983 : praep. Lyon, B.M., 603 : Ms. 788, f. 73v,20-74r,3 30-36 loc. i] Avg. serm. 46, xv, $37=$ CCSL 4I, 565,994-I004 : praep. Lyon, B.M., 603 : Ms. 788, f. 74r,II-I8 36-40 loc. j] Avg. serm. 46, xv, 38 = CCSL 4I, 565,IOIO-566,IoI6 : praep. Lyon, B.M., 603 : Ms. 788, f. 74r,23-28

2I quo eam ] qoea sic! a. corr. L 2I ne ] om. a. corr. L \| 22 hereticorum] haereticorum ed. I| 29 Cognosce et te et me. Me in caelo. te toto orbe ] Cognosce et teme in caelo, toto orbe a. corr. L Cognosce te et me : me in caelo, te in toto orbe ed. \|3 3 nisi] om. a. corr. L $\| 32$ exi] om. a. corr. L 32 hereticorum] haereticorum ed. 32 cognosce ] cognoscet $a$. corr. L $\| 33$ sequendo] $\because$ add. $\boldsymbol{F}$ in marg. L $\| 34$ pasce] pascae $a$. corr. L $\| 35$ hedos] hoedos ed. 35 hedi] hoedi ed. $\| 36$ Petro] dicitur add. ed. 36 pasce ] pascae $a$. corr. L 36 hedos] haedos $a$. corr. L hoedos ed. $\quad \| 38$ Dexteram ] $\overrightarrow{\mathrm{Ps}}$ add. $\boldsymbol{F}$ in marg. L 38 eruditos] heruditos $a$. corr. L $\| 39$ hedos] haedos $a$. corr. L hoedos ed. 39 ad prophetam ] a propheta $a$. corr. L
} 
Pierre Chambert-Protat, « Les centons augustiniens de Florus de Lyon : minutie, érudition et vulgarisation », dans Revue d'études augustiniennes et patristiques 6o/2 (2014), p. 349-379 <halshs-0I459687>

\section{Ex libro V de ciuitate Dei ${ }^{1}$}

Sancti Augustini ex libro V de ciuitate Dei

QUID IUXTA FIDEM CATHOLICAM DE DIUINA PRESCIENTIA, ET DE LIBERO ARBITRIO TENENDUM SIT,

ET QUOD DEI PROUIDENTIA ARBITRII NOBIS NON ADIMAT LIBERTATEM

Omnium conexionem seriemque causarum, qua fit omne, quod fit, ${ }^{b}$ dei summi tribuendam esse uoluntati et potestati, qui obtime et ueracissime creditur et cuncta scire, antequam fiant, et nihil inordinatum relinquere. A quo sunt omnes potestates, quamuis ab illo non sint omnium uoluntates. Ipsam itaque precipue dei summi uoluntatem, cuius potestas insuperabiliter per cuncta porrigitur, ${ }^{c}$ Cicero ita redarguere nititur, ut ${ }^{d}$ auferat diuinationem. Quam sic conatur auferre, ut neget esse scientiam futurorum eamque omnibus uiribus nullam esse omnino contendat uel in homine uel in deo nullamque rerum predictionem. Ita et dei prescientiam negat et omnem prophetiam luce clariorem conatur euertere uanis argumentationibus. ${ }^{\mathrm{e}}$ Multo sunt autem tolerabiliores, qui uel siderea fata constituunt, quam iste, qui tollit prescientiam futurorum. Nam et confiteri esse deum et negare prescium futurorum apertissima insania est. Quod et ipse cum uideret, etiam illud temptauit, quod scriptum est : Dixit insipiens in corde suo : Non est deus. ${ }^{\mathrm{f}}$ Sed quoquo modo se habeant tortuosissimae concertationes et disputationes philosophorum, nos ut confitemur summum et uerum deum, ita et uoluntatem summamqu potestatem ac prescientiam eius confitemur. Nec timemus, ne ideo non uoluntate faciamus, quod uoluntate facimus, quia id nos facturos ille presciuit, cuius prescientia falli non potest. Quod Cicero timuit, ut obpugnaret prescientiam. g Quid est ergo, quod Cicero timuit in prescientia futurorum, ut eam labefactare disputatione detestabili niteretur? Videlicet quia, si prescita sunt omnia futura, hoc ordine uenient, quo uentura esse | prescita sunt. Et si hoc ordine uenient, certus est ordo rerum prescienti deo. Et si certus est ordo rerum, certus est ordo causarum. Non enim fieri aliquid potest, quod non aliqua efficiens causa precesserit. Si autem certus est ordo causarum, quo fit omne, quod fit, fato, inquid, fiunt omnia, quae fiunt. Quod si ita est, nihil est in nostra potestate nullumque est arbitrium uoluntatis. Quod si concedimus, inquid, omnis humana uita subuertitur, frustra leges dantur, frustra obiurgationes, laudes, uituperationes, exortationes adhibentur. Neque ulla iustitia bonis premia et malis subplicia constituta sunt. Haec ergo, ne consequantur indigna et absurda et pernitiosa rebus humanis, non uult esse prescientiam

${ }^{1}$ Nous reproduisons le texte édité par Klaus ZeChiEL-EcKes d'après son unique témoin (ZeChiEL-EcKes, Klaus : «Augustinus-Rezeption im frühmittelalterlichen Lyon. Ein Quellenkritischer Beitrag zu den Beati Augustini sententiae de paredestinatione et gratia Dei et de libero hominis arbitrio. Mit edition der ungedruckten Exzerpte aus De civitate Dei (Cod. Gent 249) ", dans Quellen, Kritik, Interpretation. Festgabe zum 6o. Geburtstag von Hubert Mordek, sous la dir. de Thomas Martin Buck, Frankfurt, 1999, p. 3I-56). Nous avons seulement ajouté une numérotation des passages (de a à $\mathrm{m}$ ) et porté, dans l'apparat des sources, les références précises des préparations de la main de Florus conservées dans le manuscrit Lyon, B.M., 607.

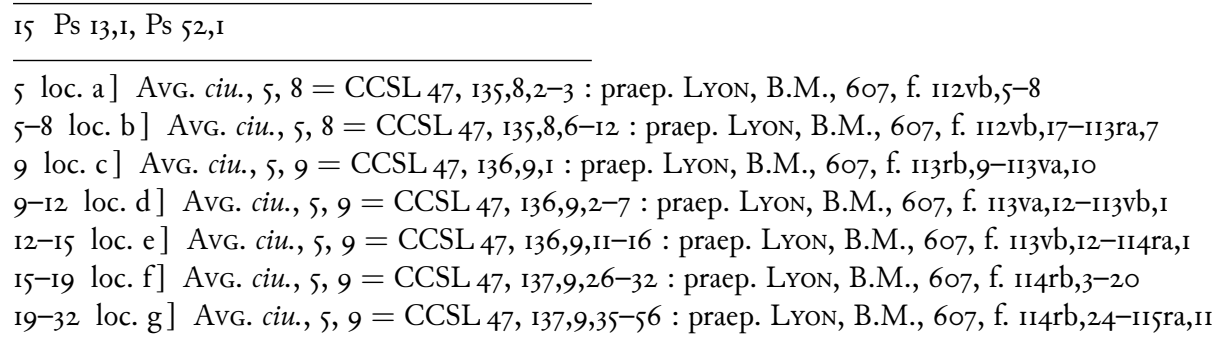


Pierre Chambert-Protat, « Les centons augustiniens de Florus de Lyon : minutie, érudition et vulgarisation », dans Revue d'études augustiniennes et patristiques 6o/2 (2014), p. 349-379 <halshs-0I459687>

futurorum. Adque in has angustias coartat animum religiosum, ut unum eligat e duobus : Aut esse aliquid in nostra uoluntate aut esse prescientiam futurorum, quoniam utrumque arbitratur esse non posse. Sed si alterum confirmabitur, alterum tolli. Si elegerimus prescientiam futurorum, tolli uoluntatis arbitrium. Si eligerimus uoluntatis arbitrium, tolli praescientia $<m>$ futurorum. Ipse itaque ${ }^{\mathrm{h}} \mathrm{ex}$ his duobus eligit liberum uoluntatis arbitrium. Quod ut confirmaretur, negauit prescientia $<\mathrm{m}>$ futurorum. Adque ita, dum uult facere liberos, facit sacrilegos. Religiosus animus utrumque eligit, utrumque confitetur et fide pietatis utrumque confirmat. Quomodo ? inquid. Nam si est prescientia futurorum, sequentur illa omnia, quae conexa sunt. Donec eo perueniatur, ut nihil sit in nostra uoluntate. Porro si est aliquid in nostra uoluntate, eisdem recursis gradibus eo peruenitur, ut non sit prescientia futurorum. Nam per illa omnia sic recurritur : Si est uoluntatis arbitrium, non omnia fato fiunt. Si non omnia fat<0> fiunt, non est omnium certus ordo causarum. Si certus ordo causarum non est, nec rerum certus est ordo prescienti deo. Quae fieri non possunt, nisi precedentibus et efficientibus causis. Si rerum ordo prescienti deo certus non est, non omnia sic ueniunt, ut ea uentura presciuit. Porro si non omnia sic ueniunt, ut ab illo uentura prescita sunt, non est, inquid, in deo prescientia omnium futurorum. Nos aduersus istos sacrilegos ausus atque impios et deum dicimus omnia scire, antequam fiant, et uoluntate nos facere, quicquid a nobis non nisi uolentibus fieri sentimus et nouimus. Omnia uero fieri fato non dicimus, immo nulla fieri fato dicimus. Quoniam fati nomen, ubi solet a loquentibus poni, id est in constitutione siderum, cum quisque conceptus aut natus est, quoniam res ipsa inaniter asseritur, nihil ualere monstramus. Ordinem autem causarum, ubi uoluntas Dei plurimum potest, neque negamus neque fati uocabulo $n<\mathrm{u}>$ ncupamus, nisi forte ut fatum a fando dictum intellegamus, id est a loquendo. Non enim abnuere possumus esse scriptum in litteris sanctis : Semel locutus est deus, duo baec audiui, quoniam potestas dei est, et tibi, domine, misericordia, qui reddes unicuique secundum opera eius. Quod enim dictum est : Semel locutus est, intellegitur immobiliter, hoc est incommutabiliter est locutus, sicut nouit incommutabiliter omnia, quae futura sunt, et quae ipse facturus est. Hac itaque ratione possemus <a fando fatum $>$ appellare, nisi hoc nomen iam in alia re soleret intellegi, quo corda hominum nolumus inclinari. Non est autem consequens, ut, si deo certus est omnium ordo causarum, ideo nihil sit in nostrae uoluntatis arbitrio. Et ipsae quippe nostrae uoluntates in causarum ordine sunt, qui certus est deo eiusque prescientia continetur. Quoniam et humanae uoluntates humanorum operum causae sunt, atque ita qui omnes rerum causas presciuit, profecto in eis causis etiam nostras uoluntates ignorare non potuit, quas nostrorum operum <causas> esse presciuit. ${ }^{\mathrm{i} S}$ Siritus ergo uitae, qui uiuificat omnia creatorque est omnis corporis et omnis creati spiritus, ipse est deus, spiritus utique non creatus. In eius uoluntate summa potestas est, quae creatorum spirituum bonas uoluntates adiuuat, malas iudicat, omnes ordinat et quibusdam tribuit potestates, quibusdam non tribuit. Sicut enim omnium naturarum creator est, ita omnium potestatum dator, non uoluntatum. Malae quippe uoluntates ab | illo non sunt, quoniam contra naturam sunt, quae ab illo est. ${ }^{j}$ Causa itaque rerum, quae facit nec fit, deus est.

65 Aliae uero causae et faciunt et fiunt, sicut sunt creati spiritus omnes, maxime rationales. Corporales autem causae, quae magis fiunt quam faciunt, non sunt inter causas efficientes annumerande, quoniam hoc possunt, quod ex ipsis faciunt spirituum uoluntates. Quo<modo $>$ igitur ordo causarum,

50-5I Ps 6I,I2-I3 (Prv 24,I2, Rm 2,6)

33-59 loc. h] Avg. ciu., 5, 9 = CCSL 47, I37,9,58-138,9,IO2 : praep. Lyon, B.M., 607, f. II5ra,15-II6rb,8

59-64 loc. i] Avg. ciu., 5, 9 = CCSL 47, I39,9,I25-I33 : praep. Lyon, B.M., 607, f. II6vb,II-II7ra,5

64-69 loc. j] Avg. ciu., 5, $9=$ CCSL 47, I39,9,138-I46 : praep. Lyon, B.M., 6o7, f. II7ra,19-II7rb,12 
Pierre Chambert-Protat, « Les centons augustiniens de Florus de Lyon : minutie, érudition et vulgarisation », dans Revue d'études augustiniennes et patristiques 6o/2 (2014), p. 349-379 <halshs-0I459687>

qui prescienti certus est deo, id efficit, ut nihil sit in nostra uoluntate, cum in ipso causarum <ordine> magnum habeant locum nostrae uoluntates? ${ }^{k}$ Quod uero negat ordinem omnium causarum esse certissimum et dei prescientiae notissimum, plus eum quam Stoici detestamur. Aut enim esse deum negat, quod quidem inducta alterius persona in libris de deorum natura facere molitus est, aut si esse confitetur deum, quem negat prescium futurorum, etiam sic nihil dicit aliud quam quod ille dixit insipiens in corde suo : Non est deus. Qui enim non est prescius omnium futurorum, non est utique deus. Quapropter et uoluntates nostrae tantum ualent, quantum deus eas ualere uoluit atque 75 presciuit. Et ideo quicquid ualent, certissime ualent, et quod facturae sunt, ipsae omnino facturae sunt, quia ualituras atque facturas ille presciuit, cuius prescientia falli non potest. ${ }^{1} \mathrm{Si}$ enim necessitas nostra illa dicenda est, quae non est in nostra potestate, sed, etiamsi nolumus, efficit, quod potest, sicut necessitas est mortis, manifestum est uoluntates nostras, quibus recte uel perperam uiuitur, sub tali necessitate non esse. Multa enim facimus, quae, si nollemus, non utique faceremus. Quo so prim<it>us pertinet ipsum uelle. Nam si uolumus, est. Si nolumus, non est. Non enim uellemus, si nollemus. Si autem illa definitur esse necessitas, secundum quam dicimus necesse esse, ut ita sit aliquid uel ita fiat, nescio cur eam timeamus, ne nobis libertatem auferat uoluntatis. Neque enim et uitam dei et prescientiam dei sub necessitate ponimus, si dicamus necesse esse deum semper uiuere et cuncta prescire. Sicut nec potestas eius minuitur, cum dicitur mori fallique non posse. Sic enim ${ }_{85}$ hoc non potest, ut potius, si posset, minoris esset utique potestatis. Recte quippe omnipotens dicitur, qui tamen falli et mori non potest. Dicitur enim omnipotens faciendo, quod uult, non patiendo, quod non uult. Quod ei si accideret, nequaquam esset omnipotens. Vnde propterea quaedam non potest, quia omnipotens est. Sic etiam cum dicimus necesse esse, ut, cum uolumus, libero uelimus arbitrio, et uerum procul dubio dicimus, et non ideo ipsum liberum arbitrium necessitati subicimus, go quae adimit libertatem. Sunt igitur nostrae uoluntates. Et ipsae faciunt, quicquid uolendo facimus, quod non fieret, si nollemus. Quicquid autem aliorum hominum uoluntate nolens quisque patitur, etiam sic uoluntas ualet, etsi non illius tamen hominis uoluntas, sed potestas dei. Nam si uoluntas tantum esset nec posset, quod uellet, potentiore uoluntate impediretur. Nec sic tamen uoluntas, nisi uoluntas esset; nec alterius, sed eius esset, qui uellet, etsi non posset implere, quod uellet. Vnde, quicquid preter suam uoluntatem patitur homo, non debet tribuere humanis uel angelicis uel cuiusquam creati spiritus uoluntatibus, sed eius potius, qui dat potestatem uolentibus. Non ergo propterea nihil est in nostra uoluntate, quia deus presciuit, qu<i>d futurum esset in nostra uoluntate. Non enim qui hoc presciuit, nihil presciuit. Porro si ille, qui praesciuit, qu $<\mathrm{i}>\mathrm{d}$ futurum esset in nostra uoluntate, non utique nihil, sed aliquid praesciuit. Profecto et illo presciente est aliquid in nostra uoluntate. Quocirca nullo modo cogimur <a>ut retenta praescientia dei tollere uoluntatis arbitrium aut retento uoluntatis arbitrio deum, quod nefas est, negare praescium futurorum. Sed utrumque amplectimur, utrumque fideliter et ueraciter confitemur. Illud, ut bene credamus, hoc, ut bene uiuamus. Male autem uiuitur, si de deo non bene creditur. Vnde et absit a nobis eius negare pres|cientiam, ut libere uelimus, quo adiuuante sumus liberi uel erimus. Proinde non frustra sunt ros leges, obiurgationes, exortationes, laudes et uituperationes. Quia et ipsas futuras esse presciuit, et ualent plurimum, quantum eas ualituras esse presciuit. Et preces ualent ad ea impetranda, quae se precantibus concessurum esse presciuit. Et iuste premia bonis factis et peccatis subplicia constituta

73 Ps i3,I, Ps 52,I

69-76 loc. k] Avg. ciu., 5, 9 = CCSL 47, I39,9,150-I40,9,162 : praep. Lyon, B.M., 607, f. II7rb,22-II7va,26 76-III loc. 1] Avg. ciu., 5, Iо = CCSL 47, I40,I0,6-I4I,IO,64 : praep. Lyon, B.M., 6o7, f. II7vb,22-II9ra,29 
Pierre Chambert-Protat, « Les centons augustiniens de Florus de Lyon : minutie, érudition et vulgarisation », dans Revue d'études augustiniennes et patristiques 60/2 (20I4), p. 349-379 <halshs-0I459687>

sunt. Neque enim ideo peccat homo, quia illum deus peccaturum esse presciuit. Immo ideo non dubitatur ipsum peccare, cum peccat, quia ille, cuius prescientia falli non potest, non fatum, non II fortunam, non aliquid aliud, sed ipsum peccaturum esse presciuit. Qui, si nolit, utique non peccat. Sed si peccare noluerit, etiam hoc ille presciuit. 
Pierre Chambert-Protat, « Les centons augustiniens de Florus de Lyon : minutie, érudition et vulgarisation », dans Revue d'études augustiniennes et patristiques 6o/2 (2014), p. 349-379 <halshs-0I459687>

\section{Beati Augustini sententiae de praedestinatione}

Apparat critique : Le centon est édité d'après l'unique témoin de la compilation des sentences augustiniennes sur la prédestination de Florus, le manuscrit GENT, U.B., 249. Nous l'avons cependant corrigé sur les préparations autographes de Florus ${ }^{2}$ conservées par le manuscrit Lyon, B.M., 608. Enfin, nous avons collationné l'édition princeps et unique de la compilation, édition publiée d'après le manuscrit de Gand et sous le nom d'Amolon par J. SiRmond en i649. Dans la marge sont données les références de ce texte tel qu'il a été réimprimé par la Patrologie latine.

Apparat comparatif : À titre purement contextuel, nous avons également collationné le centon sur ses sources, à savoir : d'une part, le manuscrit PARIs, B.n.F., lat. I0592, exemplaire des œuvres de Cyprien sur lequel Florus a travaillé par ailleurs; d'autre part, les éditions critiques des sources du centon, source directe (Avg. perseu.) et indirecte (CYPR. domin. orat.).

L codex : Lyon, B.M., 608

G codex : GeNT, U.B., 249

P codex : PARIs, B.n.F., lat. I0592

$F$ main : Florus de Lyon

Sirm. édition : Florus DE Lyon : Beati Augustini Sententiae de praedestinatione et gratia Dei, et de libero hominis arbitrio, d'après [AMOLON DE LyON] : Sancti Augustini Sententiae de praedestinatione et gratia et de libero hominis arbitrio, ante annos dccc. ex eius libris collectae, nunc uero primum editae, éd. Jacques Sirmond, Paris, 1649 (iuxta codicem G)

Aug. édition : Augustin : De dono perseuerantiae liber d'après Augustin : Aux moines d'Adrumète et de Provence, trad., annot. et introd. par Jean Chéné et Jacques Pintard (Bibliothèque Augustinienne 24), 1962

Cypr. édition : Cyprien de Carthage : De dominica oratione d'après Cyprien de Carthage: Opera, éd. M. Simonetti et C. Moreschini, t. 2 (Corpus Christianorum Series Latina 3A), Turnhout, I976, p. 87-II3

\footnotetext{
${ }^{2}$ Le manuscrit de Gand est certes le témoin du centon « mis au propre », tandis que le manuscrit de Lyon n'en est que l'ébauche. Mais le copiste du témoin est assez négligent, tandis que Florus est de son côté particulièrement scrupuleux. Ainsi de la ponctuation : celle de Gand recoupe largement celle de Lyon, mais partout où elle en diffère, c'est celle de Lyon qui s'accorde le mieux avec le sens du texte et les usages de Florus en matière de ponctuation. Nous avons donc systématiquement privilégié cette dernière.
} 
Pierre Chambert-Protat, « Les centons augustiniens de Florus de Lyon : minutie, érudition et vulgarisation », dans Revue d'études augustiniennes et patristiques 60/2 (2014), p. 349-379 <halshs-0I459687>

[II4. EX LiBRo DE PRAEDESTINATIONE SANCTORUM, itemQue de Dono Perseuerantiae, ad Prosperum et Hilarium] VII.

Oratio quae dominica nuncupatur quia eam Dominus docuit, quando oratur a sanctis, nihil paene aliud quam perseuerantia posci intellegitur. ${ }^{b}$ Dicimus enim, SANCTIFICETUR NOMEN TUUM. Non quod optemus Deo ut sanctificetur orationibus nostris, sed quod petamus ab eo, ut nomen eius sanctificetur in nobis. Ceterum, a quo Deus sanctificatur? Sed quia ipse dixit, Sancti estote quomodo et ego sanctus sum, id petimus et rogamus, ut qui in baptismo sanctificati sumus, in eo quod esse coepimus perseueremus. ${ }^{c} \mathrm{Haec}$ itaque sanctificatio, ut in nobis permaneat oramus. Et quia Dominus et iudex noster, sanato a se et uiuificato comminatur non delinquere, ne quid ei deterius fiat, hanc continuis orationibus precem facimus, hoc diebus ac noctibus postulamus, ut sanctificatio et uiuificatio quae de Dei gratia sumitur, ipsius protectione seruetur. ${ }^{\mathrm{d}}$ Quid cum dicimus, AduENIAT REGNUM TUUM. Num aliud poscimus, nisi ut ueniat et nobis, quod esse uenturum non dubitamus omnibus sanctis ? Ergo et hic qui iam sancti sunt quid orant, nisi ut in ea sanctitate quae illis data est perseuerent? Neque enim aliter ueniet Dei regnum, quod non aliis sed his qui perseuerant usque in finem certum est esse uenturum. ${ }^{\mathrm{e}}$ Fiat UOLUnTAS TUA IN CAELO ET IN TERRA. Vel quod in plerisque codicibus legitur magisque ab orantibus frequentatur, SICUT IN CAELO ET IN TERRA. Quod plerique intellegunt sicut sancti angeli, et nos faciamus uoluntatem tuam. ${ }^{\mathrm{f}} \mathrm{Vt}$ terra scilicet imitetur caelum. Id est : ut homo angelum, uel infidelis fidelem. $\mathrm{g}$ Quantalibet enim homines sanctitate praepolleant, nondum sunt aequales angelis Dei. Nondum ergo sicut in caelo, lin eis fit uoluntas Dei. ${ }^{\text {h PANEM }}$ NOSTRUM QUOTIDIANUM DA NOBIS HODIE. ${ }^{\text {i } H u n c}$ autem panem dari nobis quotidie postulamus, ne

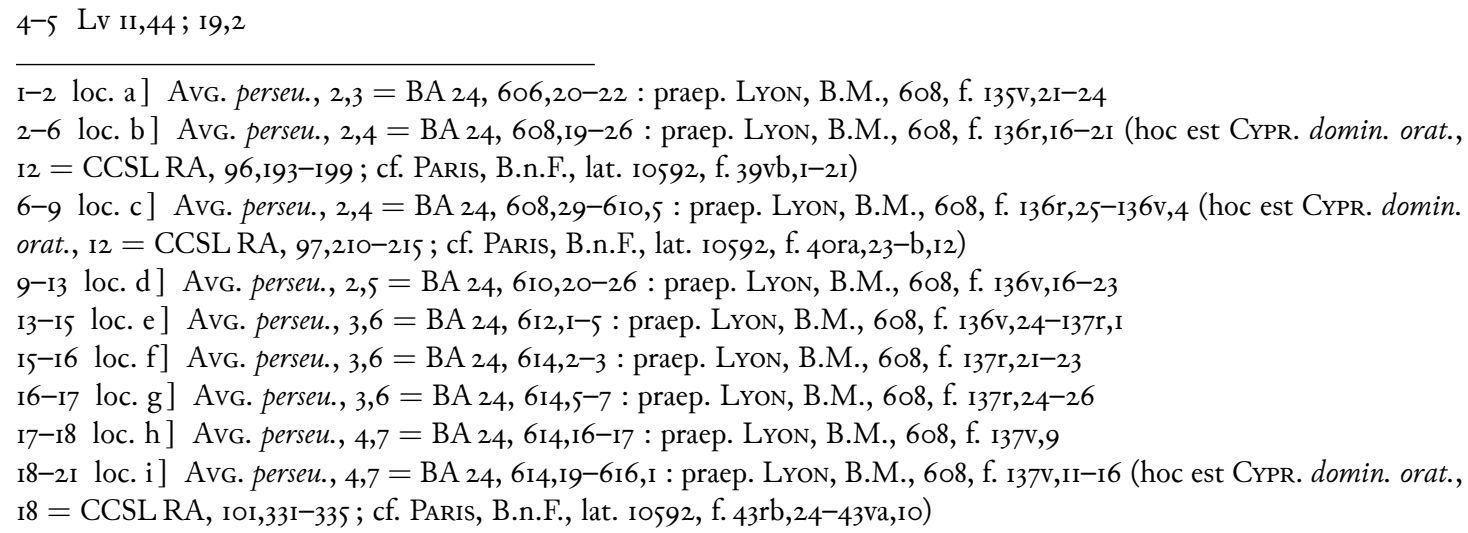

2 paene ] poene a. corr. L pene Sirm. 2 intellegitur] [cum] ... intellegatur L 2 enim ] inquit L $\|_{4}$ sanctificatur ] qui ipse sanctificat $a d d$. Sirm. 4-5 quomodo] quoniam Sirm. $\| 6$ itaque] inquit L 6 permaneat] maneat Sirm. || 8 precem] praecem G || Io Num] nam sic! G || I2 Dei regnum] regnum Dei GSirm. I2 perseuerant] perseuerent G $\| \mathrm{I} 3$ est] est add. sup. lin. L om. G I3 IN] sicut in G $\| \mathrm{I} 4 \mathrm{ab}]$ ad $a$. corr. G $\|$ I5 intellegunt] intelligunt Sirm. I| I7 Nondum] nundum sic! G || I8 QUotidianum] qotidianum a. corr. L cotidianum G I8 autem panem ] panem autem GSirm. I8 quotidie] qotidie a. corr. L cotidie G

I Oratio] oratione Aug. II 2 intellegitur] [cum] ... intelligatur Aug. 2 enim] add. Florus: inquit Aug.; om. PCypr. $\| 3 \mathrm{ab}$ eo ] a domino P $\| 4$ sanctificatur] qui ipse sanctificat add. Aug.P Cypr. 4-5 quomodo] quoniam Aug.PCypr. I| 6 itaque] add. Florus : inquit Aug.; om. PCypr. \|l 7 non] iam non PCypr. \|l 8 ac] et Aug. \|l 9 de Dei] domini P 9 ipsius] ipsi P 9 Adueniat] ueniat Aug. I| I2 ueniet] eis ueniet Aug. I2 Dei regnum] regnum Dei Aug. $\quad||$ I8 quotidie] quodtidie a. corr. $F \mathbf{P}$ 
Pierre Chambert-Protat, « Les centons augustiniens de Florus de Lyon : minutie, érudition et vulgarisation », dans Revue d'études augustiniennes et patristiques 6o/2 (2014), p. 349-379 <halshs-0I459687>

qui in Christo sumus et eucharistiam quotidie accipimus ad cibum salutis, intercedente aliquo grauiore delicto, dum abstenti et non communicantes a caelesti pane prohibemur, a Christi corpore separemur. ${ }^{j}$ DimitTe NOBIS DEBITA NOSTRA, SICUT ET NOS DIMITTIMUS DEBITORIBUS NOSTRIs. ${ }^{k}$ Quam necessarie autem, quam prouidenter et salubriter ammonemur quod peccatores sumus, qui pro peccatis rogare compellimur! Ut dum indulgentia de Deo petitur, conscientiae suae animus recordetur. $\mathrm{Ne}$ quis sibi quasi innocens placeat, et se extollendo plus pereat. Instruitur et docetur peccare se 25 quotidie, dum quotidie pro peccatis iubetur orare. Sic denique et Iohannes in epistola sua ponens dixit. Si dixerimus quia peccatum non babemus, nos ipsos decipimus, et ueritas in nobis non est. ${ }^{1}$ Iam uero cum dicunt sancti, Ne NOS INFERAS IN TEMPTATIONEM, SED LIBERA NOS A MALO : quid aliud quam ut in sanctitate perseuerent precantur? Nam profecto concesso sibi isto Dei dono, quod esse Dei donum cum ab illo poscitur, satis aperteque monstratur. Isto ergo concesso sibi dono Dei, ne inferantur in temptationem, nemo sanctorum non tenet usque in finem perseuerantiam sanctitatis. Neque enim quisquam in proposito christiano perseuerare desistit, nisi in temptationem primitus inferatur. Si ergo concedatur ei quod orat ut non inferatur : utique in sanctificatione quam Deo donante percepit, Deo donante persistit.

\footnotetext{
26 I Io $\mathrm{I}, 8$

2I loc. j] Avg. perseu., 5,8 = BA 24, 616,7-9 : praep. Lyon, B.M., 608, f. 137v,2I-22

2I-26 loc. k] Avg. perseu., 5,8 = BA 24, 616,27-618,6 : praep. Lyon, B.M., 608, f. 138r,9-17 (hoc est Cypr. domin. orat., $22=$ CCSL RA, I04,409-417; cf. PARIS, B.n.F., lat. I0592, f. 45va,17-b,15). Ultimam sententiam (sic denique - non est) primo reiecit $\boldsymbol{F}$ at accepit in fine.

26-33 loc. 1] Avg. perseu., 5,9 = BA 24, 618,8-19 : praep. Lyon, B.M., 6o8, f. 138r,I8-27
}

I9 quotidie] qotidie a. corr. L cotidie G I9 accipimus ad cibum salutis] ad cibum salutis accipimus Sirm. I9 accipimus] accepimus G 19 cibum] cybum G $\| 20$ a] ad G $\| 22$ ammonemur] admonemur Sirm. $\| 23$ compellimur] conpellimur $a$. corr. L $\| 25$ quotidie] qotidiae $a$. corr. L cotidie G 25 quotidie] qotidiae $a$. corr. L cotidie G 25 Iohannes] Ioannes Sirm. 25 epistola] epistula a. corr. L || 26 decipimus] seducimus GSirm. || $28 \mathrm{ut}$ ] add. sup. lin. G 28 concesso sibi isto] isto concesso sibi G \| 29 aperteque] aperte quae $a$. corr. L 29 dono Dei] Dei dono GSirm. \|l 30 temptationem] p. corr. L tentationem Sirm. \|l 3I in] add. sup. lin. G 3I temptationem] tentationem Sirm.

I9 eucharistiam] eius add. Cypr. I9 quotidie] quodtidie $a$. corr. $\boldsymbol{F} \mathbf{P}$ i9 accipimus ad cibum salutis ] ad cibum salutis accipimus Aug.PCypr. || 22 necessarie] necessariae P necessario Cypr. || 24 peccare se] se peccare Aug. || 25-26 ponens dixit] monet dicens PCypr. 


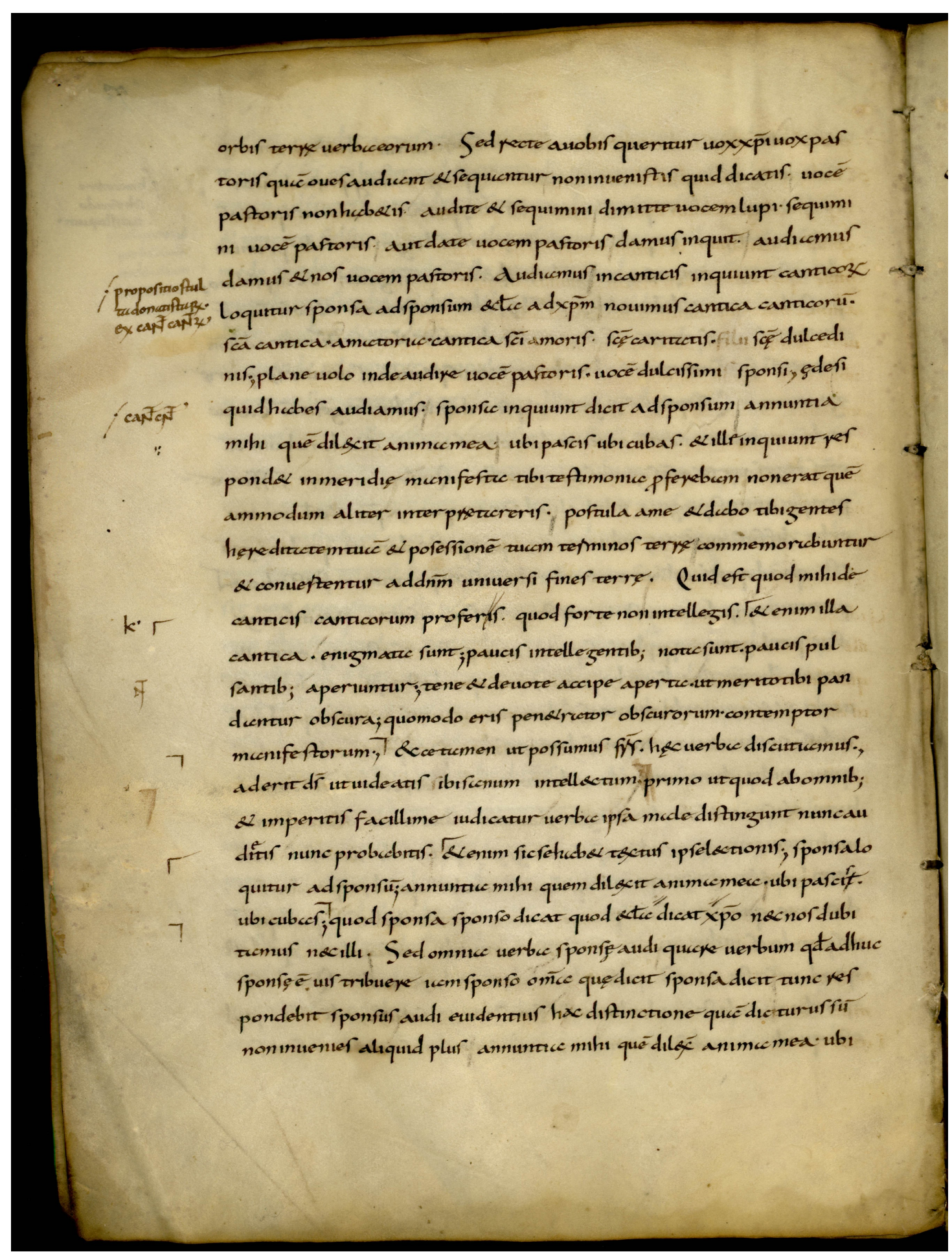

pl. I : Lyon, B.M., 788, f. 72v, dans Lyon, B.M., 603 